\title{
Success Rates of Monitoring for Healthcare Professionals with a Substance Use Disorder: A Meta-Analysis
}

\author{
Pauline M. Geuijen $\left.{ }^{1,2, *} \mathbb{(}\right)$, Sophie J. M. van den Broek ${ }^{1}$, Boukje A. G. Dijkstra ${ }^{2,3,4}{ }^{\mathbb{D}}$, Joanneke M. Kuppens ${ }^{5}$, \\ Hein A. de Haan ${ }^{2,6}{ }^{(}$, Cornelis A. J. de Jong ${ }^{2,4}$, Aart H. Schene ${ }^{1}$, Femke Atsma ${ }^{7}$ and Arnt F. A. Schellekens ${ }^{1,2}$ \\ 1 Centre for Neuroscience, Department of Psychiatry, Donders Institute for Brain, Cognition and Behaviour, \\ Radboud University Medical Center, 6500 HB Nijmegen, The Netherlands; \\ Sophie.vandenBroek@radboudumc.nl (S.J.M.v.d.B.); Aart.Schene@radboudumc.nl (A.H.S.); \\ Arnt.Schellekens@radboudumc.nl (A.F.A.S.) \\ 2 Nijmegen Institute for Scientist-Practitioners in Addiction (NISPA), 6525 HR Nijmegen, The Netherlands; \\ boukje.dijkstra@outlook.com (B.A.G.D.); H.deHaan@Tactus.nl (H.A.d.H.); \\ nispa.dejong@gmail.com (C.A.J.d.J.) \\ 3 Novadic-Kentron Addiction Care Network, 5261 LX Vught, The Netherlands \\ 4 Behavioral Science Institute, Radboud University, 6500 HE Nijmegen, The Netherlands \\ 5 Royal Dutch Medical Association (RDMA), 3528 BL Utrecht, The Netherlands; joanneke.kuppens@gmail.com \\ 6 Tactus Addiction Treatment, 7400 AD Deventer, The Netherlands \\ 7 Scientific Center for Quality of Healthcare (IQ Healthcare), Radboud Institute for Health, Radboud University \\ Medical Center, Sciences, 6500 HB Nijmegen, The Netherlands; Femke.Atsma@radboudumc.nl \\ * Correspondence: paulinegeuijen@gmail.com; Tel.: +31-243-613-489
}

\section{check for}

updates

Citation: Geuijen, P.M.; van den Broek, S.J.M.; Dijkstra, B.A.G.; Kuppens, J.M.; de Haan, H.A.; de Jong, C.A.J.; Schene, A.H.; Atsma, F.; Schellekens, A.F.A. Success Rates of Monitoring for Healthcare Professionals with a Substance Use Disorder: A Meta-Analysis. J. Clin. Med. 2021, 10, 264. https://doi.org/ $10.3390 / \mathrm{jcm} 10020264$

Received: 19 November 2020 Accepted: 31 December 2020 Published: 13 January 2021

Publisher's Note: MDPI stays neutral with regard to jurisdictional clai$\mathrm{ms}$ in published maps and institutional affiliations.

Copyright: (C) 2021 by the authors. Licensee MDPI, Basel, Switzerland. This article is an open access article distributed under the terms and conditions of the Creative Commons Attribution (CC BY) license (https:// creativecommons.org/licenses/by/ $4.0 /)$.

\begin{abstract}
In the past decades, monitoring programs have been developed for healthcare professionals with substance use disorders. We aimed to explore estimates of abstinence and work retention rates after participation in such monitoring programs. A literature search was performed using PubMed, Embase, PsycINFO, and CINAHL. Twenty-nine observational studies reporting on success rates (abstinence and work retention) of monitoring for healthcare professionals with a substance use disorder were included in the meta-analysis. Quality-effects models calculated pooled success rates and corresponding 95\%-Confidence Intervals (CI), with subgroup analyses on monitoring elements and patient characteristics. Pooled success rates were $72 \%$ for abstinence $(95 \%-\mathrm{CI}=63-80 \%)$ and $77 \%$ for work retention $(95 \%-C I=61-90 \%)$. Heterogeneity across studies was partly explained by the starting moment of monitoring, showing higher abstinence rates for studies that started monitoring after treatment completion $(79 \% ; 95 \%-\mathrm{CI}=72-85 \%)$ compared to studies that started monitoring with treatment initiation $(61 \% ; 95 \%-\mathrm{CI}=50-72 \%)$. About three-quarters of healthcare professionals with substance use disorders participating in monitoring programs are abstinent during follow-up and working at the end of the follow-up period. Due to selection and publication bias, no firm conclusions can be drawn about the effectiveness of monitoring for healthcare professionals with SUD.
\end{abstract}

Keywords: abstinence; healthcare professional; meta-analysis; monitoring; substance use disorder; work retention

\section{Introduction}

Substance Use Disorders (SUD) are a major health burden, also among healthcare providers, not only affecting their own health, but also their professional image and potentially patient safety [1,2]. Although the prevalence of SUD in healthcare professionals is estimated to be similar to that in the general population (about $10 \%$ ) $[1,3]$ they more often abuse alcohol and addictive medication, like sedatives and opioids, compared to other SUD patients $[4,5]$.

In the 1970s, the first so-called Physician Health Programs (PHPs) were initiated in the United States. PHPs aim to facilitate early identification and adequate treatment of psychiatric disorders, including SUD, among physicians [6]. Subsequently, health programs 
were established for other healthcare disciplines and in many more, mainly Western, countries across the globe $[7,8]$. The content and scope of these health programs vary widely. In the United States (US), professionals are commonly referred to inpatient and/or outpatient treatment in regular care and participate in monitoring provided by the health program [9]. In Europe, some programs mainly provide advice, others provide treatment themselves, and some offer monitoring [7]. A key difference between US health programs and some European programs (e.g., in Norway, Spain, and the United Kingdom (UK)), is that European programs encourage voluntary help seeking by offering free services and have high rates of self-referrals (45-75\%) [7]. Additionally, the UK program also guarantees confidentiality by not having any formal links with regulating authorities [10].

Monitoring offers the opportunity to follow the rehabilitation of healthcare professionals with SUD by using biological testing as an objective measure for substance use or abstinence [11]. Monitoring can be started simultaneously with treatment, as well as after successful treatment completion. In addition to biological monitoring of substance use, health programs might also monitor a participants' fitness to practice at work (by an employer or colleague) or require participation in self-help groups. Health programs usually report outcomes of rehabilitation in terms of abstinence or relapse, return to clinical practice, and/or program completion. A systematic review on rehabilitation outcomes for healthcare professionals found a variety of success rates: abstinence rates of $56 \%$ to $94 \%$ and work retention rates at the end of follow-up of $74 \%$ to $90 \%$ [12]. Previous research suggests that this variation in success rates might be influenced by both monitoring elements and participant characteristics [12,13]. Unfortunately, success rates in the systematic review were only presented as ranges per outcome and no thorough examination of the actual data was performed.

So far, there is no meta-analysis performed about success rates of monitoring for healthcare professionals with SUD. Therefore, the current meta-analysis aims to explore success rates of monitoring, using biological testing, for healthcare professionals with SUD, in terms of abstinence and work retention. Furthermore, we explored whether specific monitoring elements and/or participant characteristics explained heterogeneity in success rates across studies.

\section{Materials and Methods}

\subsection{Search Strategy and Selection Criteria}

For this meta-analysis, a review protocol was written, but not published or preregistered before the review was conducted. This protocol adopted a broad search strategy in order to maximize identification of potentially relevant papers. The search strategy, including the definition of outcome measures, was based on a set of a priori identified publications on outcomes of PHPs. The search strategy was developed by a multidisciplinary team with expertise in bibliography (medical librarian), epidemiology (P.M.G., S.J.M.v.d.B., F.A.), and addiction studies (B.A.G.D., A.F.A.S.). The search was performed on 8 December 2020 using the following databases: PubMed, Embase, PsycINFO, and CINAHL.

To be eligible, studies were required to (1) aim at adult healthcare professionals with a SUD diagnosis, (2) clearly describe their (biological) monitoring, and (3) use well-defined outcome measures in terms of abstinence (no relapse during the follow-up period) and/or work retention (working at the end of the follow-up period). Studies were excluded if (1) they concerned tobacco use disorder only, (2) no biological testing was applied, (3) the study solely reported on outcomes of care as usual, or (4) when outcomes were assessed by surveying third parties (i.e., a survey distributed among anesthesia program directors). Studies were limited to English-language research articles published in peer-reviewed journals. Details of the search strategy can be found in Table 1. 
Table 1. Search strategy.

((“Health personnel”[MeSH] OR "Medical staff"[MeSH] OR Dentist*[tiab] OR Doctor*[tiab] OR General practitioner*[tiab] OR Health personnel[tiab] OR Healthcare personnel[tiab] OR Healthcare provider*[tiab] OR Healthcare professional*[tiab] OR Medical staff[tiab] OR Nurse*[tiab] OR Nursing staff[tiab] OR Pharmacist*[tiab] OR Physician*[tiab] OR Physician assistant*[tiab])

AND

("Alcohol-related disorders"[MeSH] OR “Alcoholism"[MeSH] OR “Opioid-related disorders"[MeSH] OR "Substance-related disorders" [MeSH] OR Alcohol abuse*[tiab] OR Alcohol addict*[tiab] OR Alcohol dependen*[tiab] OR Alcohol impair*[tiab] OR Alcohol misuse[tiab] OR Alcohol use disorder*[tiab] OR Alcohol-related disorders[tiab] OR Alcoholism[tiab] OR Drug abuse*[tiab] OR Drug addict*[tiab] OR Drug

Population dependen*[tiab] OR Drug impair*[tiab] OR Drug misuse[tiab] OR Drug use disorder*[tiab] OR Opiate abuse ${ }^{*}\left[\right.$ tiab] OR Opioid abuse*[tiab] OR Opiate addict* ${ }^{*}[$ tiab] OR Opioid addict*[tiab] OR Opiate dependen*[tiab] OR Opioid dependen*[tiab] OR Opiate impair*[tiab] OR Opioid impair*[tiab] OR Opiate misuse[tiab] OR Opioid misuse[tiab] OR Opioid-related disorders[tiab] OR Substance abuse ${ }^{*}[$ tiab] OR Substance addict*[tiab] OR Substance dependen*[tiab] OR Substance impair*[tiab] OR Substance misuse[tiab] OR Substance use disorder*[tiab] OR Substance-related disorders[tiab]))

OR

("Professional impairment"[MeSH] OR Dentist impair*[tiab] OR Doctor impair*[tiab] OR Nurse impair*[tiab] OR Pharmacist impair*[tiab] OR Physician impair*[tiab] OR Physician assistant impair*[tiab] OR Professional impairment[tiab])

\section{AND}

("Health services" [MeSH] OR “Occupational health"[MeSH] OR “Mental disorders"[MeSH] OR “Referral and consultation" [MeSh] OR Employee assistance program*[tiab] OR Employee health service*[tiab] OR Health agenc*[tiab] OR Health program*[tiab] OR Health service*[tiab] OR Occupational health[tiab] OR Occupational health service*[tiab] OR Mental disorders[tiab] OR Referral and consultation[tiab]) OR

Intervention

("Biological monitoring"[MeSH] OR "Mental health recovery"[MeSH] OR "Psychiatric rehabilitation"[MeSH] OR Biological monitor*[tiab] OR Mental health rehabilitation[tiab] OR Mental health recovery[tiab] OR Physiologic monitor*[tiab] OR Psychiatric rehabilitation[tiab] OR Psychosocial rehabilitation[tiab] OR Recover*[tiab])

OR

("Self-help groups"[MeSH] OR Self-help group*[tiab] OR Support group*[tiab] OR Alcoholics anonym*[tiab] OR Narcotics anonym*[tiab])

\section{AND}

(“Outcome assessment, health care" $[\mathrm{MeSH}] \mathrm{OR}$ "Program evaluation"[MeSH] OR “Treatment outcome"[MeSH] OR Outcome assessment ${ }^{*}[$ tiab] OR Outcome measure*[tiab] OR Program effect*[tiab] OR Program evaluation[tiab] OR Treatment effect*[tiab] OR Treatment failure*[tiab] OR Treatment outcome*[tiab] OR Recovery rate*[tiab] OR Rehabilitation outcome*[tiab]) OR

Outcome ("Alcohol abstinence"[MeSH] OR "Recurrence"[MeSH] OR Abstinence[tiab] OR Alcohol abstinence[tiab] OR Drug abstinence[tiab] OR Opioid abstinence[tiab] OR Substance use abstinence[tiab] OR Recurrence[tiab] OR Relapse*[tiab])

OR

("Return to work"[MeSH] OR “Work performance"[MeSH] OR Job perform*[tiab] OR Job retention[tiab] OR Return to work[tiab] OR Work perform*[tiab] OR Work resum*[tiab] OR Work retention[tiab])

* This strategy is related to the PubMed search. Very similar versions were used to search Embase, PsycINFO, and CINAHL, but adapted for the specific search terms used in these databases.

\subsection{Study Selection, Data Extraction, and Quality Assessment}

A flow chart of the study selection procedure is provided in Figure 1. First, duplicates were removed, using Rayyan software (Qatar Computing Research Institute, Doha, Qatar, 2017) for citation screening [14]. Next, three authors (P.M.G., S.J.M.v.d.B., and B.A.G.D.) screened 5907 unique titles and abstracts on the selection criteria mentioned above. Discrepancies in the identified eligible records were discussed until consensus was reached. When in doubt, records moved on to the next phase of assessing the eligibility, based on the full-text articles. Full-text assessment of 94 remaining records was performed by two authors (P.M.G. and S.J.M.v.d.B.). Discrepancies were discussed until consensus was reached. This resulted in 29 studies eligible for the meta-analysis, published in 24 articles. 
Next, data-extraction was performed by one researcher (P.M.G.). The data of each study was documented in Microsoft Excel 2016, which was subsequently checked by a second researcher (S.J.M.v.d.B).

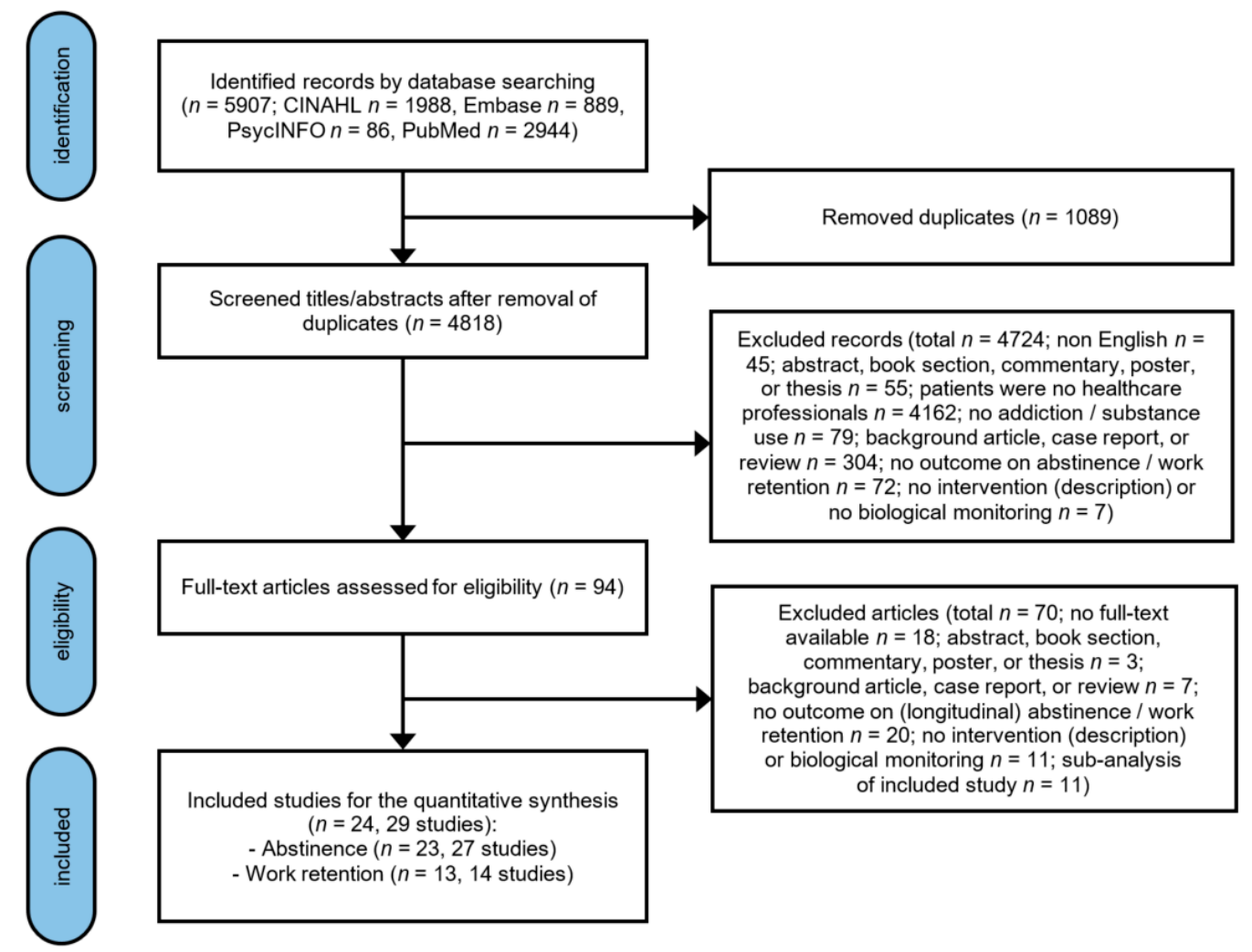

Figure 1. PRISMA flowchart of the selection of studies.

Extracted information included study characteristics: name of first author, year of publication, country (state) of first author, design of the study, time frame of the study, number of included subjects, percentage of males, type of healthcare professional, type of substance use, and source of referral. In addition, characteristics of monitoring were summarized: name of the health program, recommended type of treatment, starting moment of monitoring, type of biological testing, monitoring at work, and additional agreements. Finally, the outcomes of monitoring programs were extracted: percentage of abstinence and work retention specified with the (exact or range of) duration of follow-up. Since the information was not always presented in the same manner, we categorized monitoring elements and participant characteristics in order to perform subgroup analyses: program elements (biological, at work, and additional agreements; biological and additional agreements; biological and at work; biological), starting moment of monitoring (before treatment; after treatment; unknown), duration of follow-up (less than 2 years; 2 to 5 years; more than 5 years; other duration), gender (more than $50 \%$ males; other or unknown), type of healthcare professional (more than $50 \%$ physicians; other or mixed), and type of substance use (more than $50 \%$ alcohol; more than $50 \%$ opioids; mixed or unknown).

All included studies were assessed on their quality in order to account for study quality in the meta-analysis. The initial assessment was performed by one researcher (P.M.G.), and subsequently checked by a second researcher (S.J.M.v.d.B.). The Health States Quality Index [15] was used to assess study quality. Assessment parameters include a clear definition of the target population and observation period (yes or no), use of diagnostic criteria (diagnostic system or symptom based/not specified), method of case selection (attempting all cases, convenience sampling, or not specified), type of outcome assessment (administered interview, register/case record, or not specified), size of the study area (broad, small, or not specified), and type of prevalence measure (exact follow-up duration, average 
follow-up duration, or range of follow-up duration). The quality index of each study is calculated as the total quality score of that study divided by the maximum total quality score, see Table A1. The instrument was slightly adjusted for a good fit to our meta-analysis. The higher the score, the higher the study quality. We report our study in accordance with the Preferred Reporting Items for Systematic reviews and Meta-Analyses (PRISMA) and the proposal for reporting Meta-analyses of Observational Studies in Epidemiology (MOOSE) were applicable, see Table S1 [16,17].

\subsection{Data-Analysis}

Statistical analyses were performed using MetaXL (EpiGear International Pty Ltd., Sunrise Beach, Australia, version 5.3) within Microsoft Excel 2016 [15,18]. For every study, the total number of participants, the number of participants with a successful outcome (abstinence or work retention), and the quality index were entered in MetaXL. Quality-effects models were used in order to address heterogeneity caused by differences in study quality. The quality-effects model is a modified version of the fixed-effects inverse variance method and gives greater weight to the studies that were judged as being of high quality [19]. The models were applied to analyze the data and calculate pooled abstinence and work retention rates, and accompanying 95\%-Confidence Intervals (CI).

The heterogeneity assumption was assessed by Cochrane's Q-test (which verifies the presence of heterogeneity) and I2 statistic (which shows the amount of heterogeneity between studies). A significant Q-test $(p<0.10)$ and an I2 $>50 \%$ indicated the presence of substantial heterogeneity. Subgroup analyses were explored by stratifying the data on monitoring elements (start of monitoring, type of monitoring, and duration of followup) and participant characteristics (gender, type of healthcare professional, and type of substance use).

Publication bias was assessed using the Doi plot and Luis Furuya-Kanamori asymmetry (LFK) index. In the case of a symmetric shape, no publication bias is indicated. In case of an asymmetric shape, publication bias is indicated. An LFK index within -1 and +1 indicates no publication bias, an LFK of -1 to -2 or +1 to +2 minor asymmetry, and an LFK of $<-2$ or $>+2$ major asymmetry [15].

\section{Results}

\subsection{Description of the Included Studies}

The study and monitoring characteristics of the 29 included studies (out of 24 articles) are summarized in Tables 2 and 3. Studies were published between 1982 and 2020 and mainly conducted in the United States, one in Canada, one in Australia, and one in Spain. The design of most studies was observational (either retrospective or prospective data collection). One study had a descriptive design (survey among healthcare professionals engaged in a monitoring program), and one performed an experiment (single-arm multisite, open label study of injectable naltrexone in healthcare professionals with opioid dependence). None of the included studies used randomized controlled trial or quasiexperimental designs. 
Table 2. Study characteristics.

\begin{tabular}{|c|c|c|c|c|c|c|c|}
\hline $\begin{array}{l}\text { Year, Study } \\
\text { Country (State) }\end{array}$ & Design & $\begin{array}{l}\text { Time } \\
\text { Frame }\end{array}$ & $\begin{array}{l}\text { Subjects } \\
\text { (N) }\end{array}$ & Males (\%) & $\begin{array}{l}\text { Type of Healthcare Professional } \\
(\%)\end{array}$ & $\begin{array}{l}\text { Type of Substance Use } \\
(\%)\end{array}$ & Source of Referral (\%) \\
\hline $\begin{array}{l}\text { 1982, Herrington et al. [20] } \\
\text { US (Wisconsin) }\end{array}$ & $\begin{array}{l}\text { retrospective } \\
\text { review }\end{array}$ & 1979-1982 & 40 & 95 & $\begin{array}{l}\text { general practitioner (28); } \\
\text { anesthesiologist (13); psychiatrist (10); } \\
\text { internal medicine (8); dentist (8); } \\
\text { obstetrics-gynecology (8); surgeon (5); } \\
\text { other (20) }\end{array}$ & $\begin{array}{l}\text { alcohol (58); narcotics (38); } \\
\text { other (5) }\end{array}$ & $\begin{array}{l}\text { coworker (63); family } \\
\text { member (18); legal system } \\
\text { (13); self-referral (8) }\end{array}$ \\
\hline $\begin{array}{l}\text { 1984, Washton et al. [21] } \\
\text { US (New York; New } \\
\text { Jersey) }\end{array}$ & $\begin{array}{l}\text { retrospective } \\
\text { review }\end{array}$ & 1979-1981 & 15 & 100 & physician (100) & opioids (100) & - \\
\hline $\begin{array}{l}\text { 1985, Crowley [22] } \\
\text { US (Colorado) }\end{array}$ & $\begin{array}{l}\text { prospective } \\
\text { descriptive }\end{array}$ & - & 15 & 100 & $\begin{array}{l}\text { physician (60); dentist (33); } \\
\text { veterinarian (7) }\end{array}$ & - & $\begin{array}{l}\text { licensing board (40); hospital } \\
\text { or coworkers ( } 33 \text { ); family } \\
\text { member (7); treatment } \\
\text { provider (7); self-referral (13) }\end{array}$ \\
\hline $\begin{array}{l}\text { 1987, Shore [23] } \\
\text { US (Oregon) }\end{array}$ & $\begin{array}{l}\text { retrospective } \\
\text { review }\end{array}$ & 1977-1985 & 25 & - & physician (100) & - & - \\
\hline $\begin{array}{l}\text { 1991, Pelton \& Ikeda [24] } \\
\text { US (California) }\end{array}$ & $\begin{array}{l}\text { retrospective } \\
\text { review }\end{array}$ & 1980-1990 & 51 & - & anesthesiologist (100) & opioids (49) & - \\
\hline $\begin{array}{l}\text { 1992, Gallegos et al. [25] } \\
\text { US (Georgia) }\end{array}$ & $\begin{array}{l}\text { retrospective } \\
\text { review }\end{array}$ & 1982-1992 & 100 & 92 & $\begin{array}{l}\text { family and general practitioner (23); } \\
\text { surgeon (22); anesthesiologist (17); } \\
\text { psychiatrist (15); internal medicine } \\
\text { (12); emergency medicine (4); } \\
\text { pediatrician (3); radiologist (1); } \\
\text { dermatologist (1); occupational } \\
\text { medicine (1); rehabilitation } \\
\text { medicine (1) }\end{array}$ & $\begin{array}{l}\text { alcohol (71); cocaine (21); } \\
\text { meperidine hydrochloride (19); } \\
\text { diazepam (18); marijuana (17); } \\
\text { percodan (12); fentanyl citrate } \\
\text { (11); codeine sulfate (9); } \\
\text { amphetamine (7) }\end{array}$ & - \\
\hline $\begin{array}{l}\text { 1994, Roy [26] } \\
\text { US (Louisiana) }\end{array}$ & $\begin{array}{l}\text { retrospective } \\
\text { review }\end{array}$ & $>1989$ & 37 & 89 & $\begin{array}{l}\text { physician (68); dentist (16); } \\
\text { pharmacist (5); veterinarian (3); } \\
\text { other (8) }\end{array}$ & $\begin{array}{l}\text { prescription drug (43); alcohol } \\
\text { (27); polysubstance (16); } \\
\text { cocaine (14) }\end{array}$ & - \\
\hline
\end{tabular}


Table 2. Cont.

\begin{tabular}{|c|c|c|c|c|c|c|c|}
\hline $\begin{array}{l}\text { Year, Study } \\
\text { Country (State) }\end{array}$ & Design & $\begin{array}{l}\text { Time } \\
\text { Frame }\end{array}$ & $\begin{array}{l}\text { Subjects } \\
\text { (N) }\end{array}$ & Males (\%) & $\begin{array}{l}\text { Type of Healthcare Professional } \\
\text { (\%) }\end{array}$ & $\begin{array}{l}\text { Type of Substance Use } \\
(\%)\end{array}$ & Source of Referral (\%) \\
\hline $\begin{array}{l}\text { 1996, Nelson et al. (1) [27] } \\
\text { US (Oregon) }\end{array}$ & $\begin{array}{l}\text { retrospective } \\
\text { review }\end{array}$ & 1990-1992 & 56 & 91 & \multirow{2}{*}{$\begin{array}{l}\text { surgery (59); internal medicine (32); } \\
\text { family practitioner (21); emergency } \\
\text { medicine (7); anesthesiology (6); } \\
\text { pathology (4); pediatrician (4); } \\
\text { obstetrics-gynecology (3); psychiatry } \\
\text { (2); neurology (2); dermatology (1); } \\
\text { radiology (1); unknown (1) }\end{array}$} & $\begin{array}{l}\text { alcohol ( } 75) \text {; opioids and cocaine } \\
(21) ; \text { amphetamines and } \\
\text { sedatives (4) }\end{array}$ & $\begin{array}{l}\text { self-referral (15); immediate } \\
\text { contact (39); third party (46) }\end{array}$ \\
\hline $\begin{array}{l}\text { 1996, Nelson et al. (2) [27] } \\
\text { US (Oregon) }\end{array}$ & $\begin{array}{l}\text { retrospective } \\
\text { review }\end{array}$ & 1990-1992 & 41 & 90 & & $\begin{array}{l}\text { alcohol (87); opioids and cocaine } \\
\text { (8); amphetamines and } \\
\text { sedatives (5) }\end{array}$ & $\begin{array}{l}\text { self-referral (7); immediate } \\
\text { contact (15); third party (73); } \\
\text { unknown (5) }\end{array}$ \\
\hline $\begin{array}{l}\text { 1999, Paris \& Canavan (1) } \\
\text { [29] } \\
\text { US (New Jersey) }\end{array}$ & $\begin{array}{l}\text { retrospective } \\
\text { review }\end{array}$ & 1982-1994 & 32 & - & $\begin{array}{l}\text { anesthesiologist (59); anesthesiology } \\
\text { residents (41) }\end{array}$ & opioids (78) & - \\
\hline $\begin{array}{l}\text { 1999, Paris \& Canavan (2) } \\
\text { [29] } \\
\text { US (New Jersey) }\end{array}$ & $\begin{array}{l}\text { retrospective } \\
\text { review }\end{array}$ & 1982-1994 & 36 & - & physician (75); resident (25) & opioids (42) & - \\
\hline $\begin{array}{l}\text { 2005, Domino et al. [31] } \\
\text { US (Washington) }\end{array}$ & $\begin{array}{l}\text { retrospective } \\
\text { cohort }\end{array}$ & 1991-2001 & 292 & 84 & $\begin{array}{l}\text { physician (79); physician assistant } \\
\text { (11); veterinarian (5); osteopath (2); } \\
\text { dentist/dental surgeon (1); } \\
\text { podiatrist/pharmacist (1) }\end{array}$ & $\begin{array}{l}\text { alcohol (56); opioids ( } 32) \text {; } \\
\text { cocaine (3); benzodiazepines (2); } \\
\text { other (7) }\end{array}$ & - \\
\hline $\begin{array}{l}\text { 2005, Ganley et al. (1) [32] } \\
\text { US (North Carolina) }\end{array}$ & $\begin{array}{l}\text { retrospective } \\
\text { review }\end{array}$ & 1991-2001 & 233 & 87 & physician (100) & $\begin{array}{l}\text { alcohol (50); opioids (25); } \\
\text { polysubstance (16); other (8) }\end{array}$ & $\begin{array}{l}\text { licensing board; hospital; } \\
\text { coworker; family member; } \\
\text { self-referral }\end{array}$ \\
\hline
\end{tabular}


Table 2. Cont.

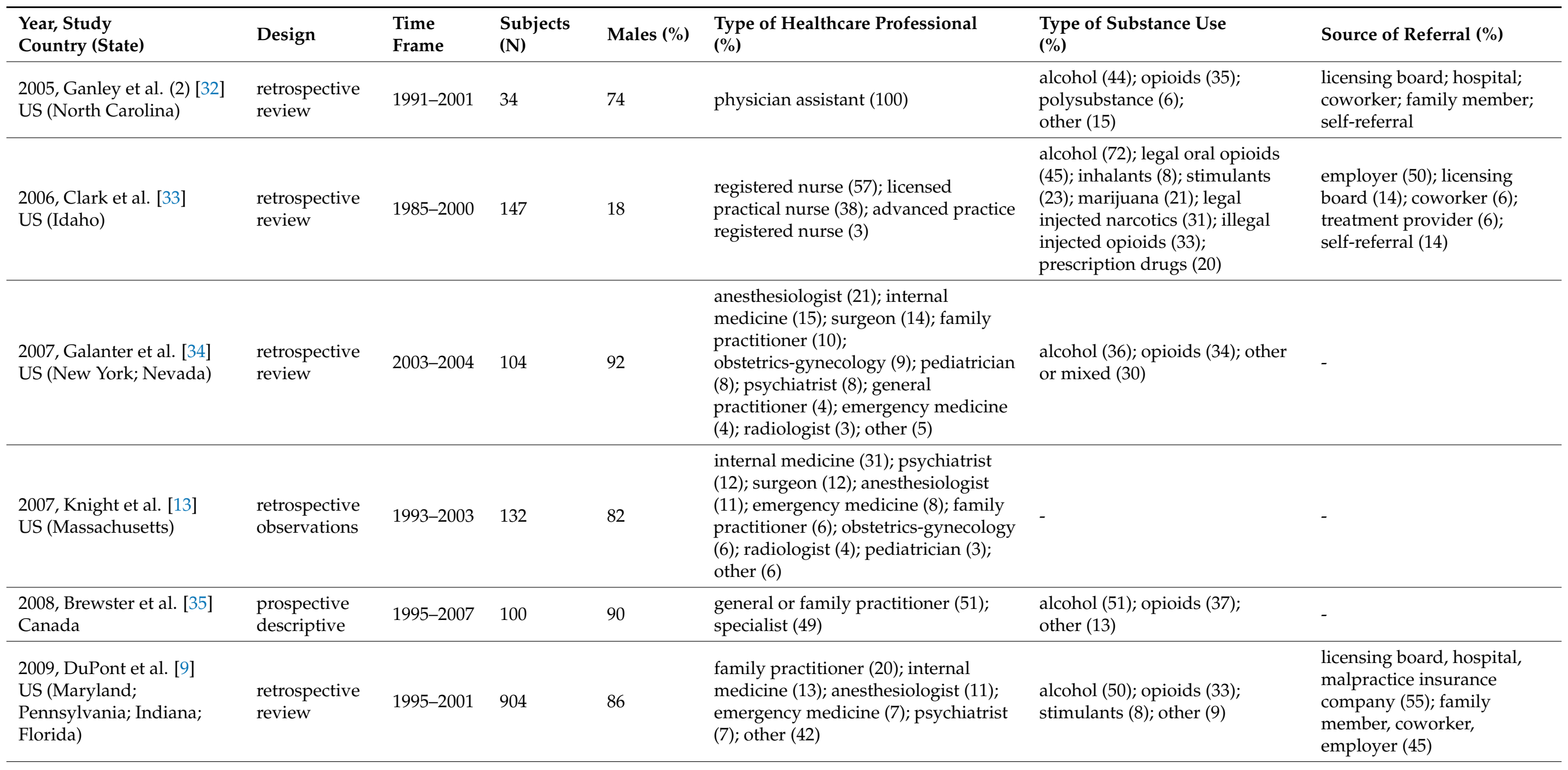


Table 2. Cont.

\begin{tabular}{|c|c|c|c|c|c|c|c|}
\hline $\begin{array}{l}\text { Year, Study } \\
\text { Country (State) }\end{array}$ & Design & $\begin{array}{l}\text { Time } \\
\text { Frame }\end{array}$ & $\begin{array}{l}\text { Subjects } \\
\text { (N) }\end{array}$ & Males (\%) & $\begin{array}{l}\text { Type of Healthcare Professional } \\
(\%)\end{array}$ & $\begin{array}{l}\text { Type of Substance Use } \\
(\%)\end{array}$ & Source of Referral (\%) \\
\hline $\begin{array}{l}\text { 2009, Fogger \& } \\
\text { Mc-Guinness (1) [36] } \\
\text { US (Alabama) }\end{array}$ & $\begin{array}{l}\text { cross- } \\
\text { sectional } \\
\text { survey }\end{array}$ & - & 127 & - & $\begin{array}{l}\text { registered nurse (77); licensed } \\
\text { practical nurse (13); advanced practice } \\
\text { registered nurse (8) }\end{array}$ & \multirow{2}{*}{ opioids (36) } & - \\
\hline $\begin{array}{l}\text { 2009, Fogger \& } \\
\text { Mc-Guinness (2) [36] } \\
\text { US (Alabama) }\end{array}$ & $\begin{array}{l}\text { cross- } \\
\text { sectional } \\
\text { survey }\end{array}$ & - & 45 & - & $\begin{array}{l}\text { registered nurse }(78) \text {; licensed } \\
\text { practical nurse (18); advanced practice } \\
\text { registered nurse (4) }\end{array}$ & & - \\
\hline $\begin{array}{l}\text { 2013, Angres et al. [38] } \\
\text { US (Illinois) }\end{array}$ & $\begin{array}{l}\text { prospective } \\
\text { cohort }\end{array}$ & - & 116 & 68 & $\begin{array}{l}\text { physician (48); nurse (24); pharmacist } \\
\text { (18); dentist (7); optometrist (1); } \\
\text { physician assistant (1); other (1) }\end{array}$ & - & licensing board (100) \\
\hline $\begin{array}{l}\text { 2013, Cross et al. [39] } \\
\text { US (Illinois) }\end{array}$ & $\begin{array}{l}\text { prospective } \\
\text { descriptive }\end{array}$ & 1994-2011 & 116 & 78 & pharmacist (100) & $\begin{array}{l}\text { oral opioids (71); alcohol (22); } \\
\text { illegal drugs (9); stimulants (8); } \\
\text { injected opioids (3) }\end{array}$ & - \\
\hline $\begin{array}{l}\text { 2017, Earley et al. [40] } \\
\text { US (Georgia) }\end{array}$ & $\begin{array}{l}\text { single-arm } \\
\text { multisite, } \\
\text { open label }\end{array}$ & 2009-2012 & 38 & 18 & $\begin{array}{l}\text { nurse (79); physician (11); pharmacist } \\
\text { (3); other (8) }\end{array}$ & opioids (100) & - \\
\hline $\begin{array}{l}\text { 2020, Bruguera et al. [41] } \\
\text { Spain }\end{array}$ & $\begin{array}{l}\text { prospective } \\
\text { descriptive }\end{array}$ & 2008-2016 & 126 & 60 & $\begin{array}{l}\text { family practitioner (17); psychiatrist } \\
\text { (9); anesthesiologist (9); pediatrician } \\
\text { (6); orthopedic surgeons (6); internal } \\
\text { medicine (3); resident (4); other (47) }\end{array}$ & $\begin{array}{l}\text { alcohol (63); sedatives, } \\
\text { hypnotics, anxiolytics (15); } \\
\text { opioids (7); stimulants (6); } \\
\text { cannabis (4); cocaine (2); } \\
\text { mixed (3) }\end{array}$ & $\begin{array}{l}\text { self-referral (75); coworker } \\
\text { or family member }(20) \text {; } \\
\text { other }(6)\end{array}$ \\
\hline
\end{tabular}


Table 3. Characteristics of monitoring.

\begin{tabular}{|c|c|c|c|c|c|c|c|c|}
\hline \multirow[b]{2}{*}{ Year, Study } & \multirow{2}{*}{$\begin{array}{l}\text { Name of the } \\
\text { Health } \\
\text { Program }\end{array}$} & \multirow[b]{2}{*}{$\begin{array}{l}\text { Recommended } \\
\text { Type of } \\
\text { Treatment }\end{array}$} & \multicolumn{4}{|c|}{ Monitoring Elements } & \multicolumn{2}{|c|}{ Monitoring Outcomes } \\
\hline & & & $\begin{array}{l}\text { Start of } \\
\text { Monitoring }\end{array}$ & $\begin{array}{l}\text { Biological } \\
\text { Monitoring }\end{array}$ & $\begin{array}{l}\text { Monitoring at } \\
\text { Work }\end{array}$ & Other Agreements & $\begin{array}{l}\text { Follow-Up: } \\
\text { \% Abstinence }\end{array}$ & $\begin{array}{l}\text { Follow-Up: } \\
\text { \% Work } \\
\text { Retention }\end{array}$ \\
\hline $\begin{array}{l}\text { 1982, Herrington } \\
\text { et al. [20] }\end{array}$ & $\begin{array}{l}\text { Impaired } \\
\text { Physician } \\
\text { Treatment } \\
\text { Program }\end{array}$ & inpatient (95\%) & $\begin{array}{l}\text { after treatment } \\
\text { completion }\end{array}$ & urine & yes & $\begin{array}{l}\text { participate in Alcoholics or } \\
\text { Narcotics Anonymous groups, } \\
\text { attendance of local meetings in the } \\
\text { community, and attendance of weekly } \\
\text { sessions of Milwaukee Doctors in } \\
\text { Alcoholics Anonymous }\end{array}$ & $\begin{array}{l}0 \text { to } 3 \text { years: } \\
68 \% \text { no relapse }\end{array}$ & $\begin{array}{l}0 \text { to } 3 \text { years: } \\
78 \% \text { working }\end{array}$ \\
\hline $\begin{array}{l}\text { 1984, Washton } \\
\text { et al. [21] }\end{array}$ & $\begin{array}{l}\text { Regent or Fair } \\
\text { Oaks Hospital }\end{array}$ & $\begin{array}{l}\text { inpatient } \\
4-10 \text { weeks }\end{array}$ & $\begin{array}{l}\text { after treatment } \\
\text { completion }\end{array}$ & urine & & $\begin{array}{l}\text { pharmacotherapy with naltrexone, } \\
\text { group therapy, individual therapy, and } \\
\text { family/couples therapy }\end{array}$ & $\begin{array}{l}0 \text { to } 1.5 \text { years: } \\
87 \% \text { no relapse }\end{array}$ & $\begin{array}{l}0 \text { to } 1.5 \text { years: } \\
87 \% \text { working }\end{array}$ \\
\hline 1985, Crowley [22] & Halsted Clinic & outpatient & $\begin{array}{l}\text { with treatment } \\
\text { initiation }\end{array}$ & urine & - & counseling sessions & $\begin{array}{l}2 \text { years (average): } \\
47 \% \text { no relapse }\end{array}$ & - \\
\hline 1987, Shore [23] & $\begin{array}{l}\text { rehabilitation } \\
\text { program }\end{array}$ & - & - & urine & - & $\begin{array}{l}\text { long term supervision by the } \\
\text { medical board }\end{array}$ & - & $\begin{array}{l}0 \text { to } 8 \text { years: } \\
75 \% \text { working }\end{array}$ \\
\hline $\begin{array}{l}\text { 1992, Gallegos } \\
\text { et al. [25] }\end{array}$ & $\begin{array}{l}\text { Georgia } \\
\text { Impaired } \\
\text { Physicians } \\
\text { Program } \\
\text { continuing } \\
\text { care program }\end{array}$ & $\begin{array}{l}\text { Georgia } \\
\text { Impaired } \\
\text { Physicians } \\
\text { Treatment } \\
\text { Program }\end{array}$ & $\begin{array}{l}\text { after treatment } \\
\text { completion }\end{array}$ & urine & - & $\begin{array}{l}\text { primary care physician attends to their } \\
\text { medical needs, recovery mentor, five } \\
\text { Alcoholics or Narcotics Anonymous } \\
\text { meetings per week, and one Caduceus } \\
\text { Club meeting per week }\end{array}$ & $\begin{array}{l}\text { more than } 5 \text { years: } \\
77 \% \text { no relapse }\end{array}$ & $\begin{array}{l}\text { more than } \\
5 \text { years: } \\
91 \% \text { working }\end{array}$ \\
\hline 1994, Roy [26] & $\begin{array}{l}\text { reentry } \\
\text { monitoring }\end{array}$ & - & $\begin{array}{l}\text { after treatment } \\
\text { completion }\end{array}$ & urine & - & group therapy & $\begin{array}{l}2 \text { years (average): } \\
81 \% \text { no relapse } 8 \% \\
\text { brief relapse }\end{array}$ & $\begin{array}{l}2 \text { years } \\
\text { (average): } \\
95 \% \text { working }\end{array}$ \\
\hline $\begin{array}{l}\text { 1996, Nelson et al. } \\
\text { (1) [27] }\end{array}$ & $\begin{array}{l}\text { Diversion } \\
\text { Program }\end{array}$ & $\begin{array}{l}\text { inpatient } \\
\text { and/or } \\
\text { outpatient }\end{array}$ & $\begin{array}{l}\text { after treatment } \\
\text { completion }\end{array}$ & urine & - & group therapy & $\begin{array}{l}1.5 \text { years (average): } \\
86 \% \text { no relapse }\end{array}$ & - \\
\hline
\end{tabular}


Table 3. Cont.

\begin{tabular}{|c|c|c|c|c|c|c|c|c|}
\hline \multirow[b]{2}{*}{ Year, Study } & \multirow{2}{*}{$\begin{array}{l}\text { Name of the } \\
\text { Health } \\
\text { Program }\end{array}$} & \multirow{2}{*}{$\begin{array}{l}\text { Recommended } \\
\text { Type of } \\
\text { Treatment }\end{array}$} & \multirow[b]{2}{*}{$\begin{array}{l}\text { Start of } \\
\text { Monitoring }\end{array}$} & \multirow[b]{2}{*}{$\begin{array}{l}\text { Biological } \\
\text { Monitoring }\end{array}$} & \multicolumn{2}{|c|}{ Monitoring Elements } & \multicolumn{2}{|c|}{ Monitoring Outcomes } \\
\hline & & & & & $\begin{array}{l}\text { Monitoring at } \\
\text { Work }\end{array}$ & Other Agreements & $\begin{array}{l}\text { Follow-Up: } \\
\text { \% Abstinence }\end{array}$ & $\begin{array}{l}\text { Follow-Up: } \\
\text { \% Work } \\
\text { Retention }\end{array}$ \\
\hline $\begin{array}{l}\text { 1996, Nelson et al. } \\
\text { (2) [27] }\end{array}$ & $\begin{array}{l}\text { Probationary } \\
\text { Program }\end{array}$ & $\begin{array}{l}\text { inpatient } \\
\text { and/or } \\
\text { outpatient }\end{array}$ & $\begin{array}{l}\text { after treatment } \\
\text { completion }\end{array}$ & urine & - & group therapy & $\begin{array}{l}2.3 \text { years (average): } \\
78 \% \text { no relapse }\end{array}$ & - \\
\hline $\begin{array}{l}\text { 1997, Roth } \\
\text { et al. [28] }\end{array}$ & $\begin{array}{l}\text { special } \\
\text { treatment } \\
\text { program }\end{array}$ & $\begin{array}{l}\text { inpatient } \\
\text { and/or } \\
\text { outpatient }\end{array}$ & $\begin{array}{l}\text { with treatment } \\
\text { initiation }\end{array}$ & urine & - & $\begin{array}{l}\text { pharmacotherapy with naltrexone for } \\
6 \text { months }\end{array}$ & $\begin{array}{l}1.8 \text { years (average): } \\
60 \% \text { no relapse }\end{array}$ & $\begin{array}{l}1.8 \text { years } \\
\text { (average): } \\
60 \% \text { working }\end{array}$ \\
\hline $\begin{array}{l}\text { 1999, Paris \& } \\
\text { Canavan (1) [29] }\end{array}$ & $\begin{array}{l}\text { Physician } \\
\text { Health } \\
\text { Program }\end{array}$ & - & $\begin{array}{l}\text { after treatment } \\
\text { completion }\end{array}$ & urine & - & $\begin{array}{l}\text { participate in an aftercare group } \\
\text { for } 1 \text { year, monthly face-to-face } \\
\text { appointment with PHP employee, and } \\
\text { attendance of Alcoholics } \\
\text { Anonymous meetings }\end{array}$ & $\begin{array}{l}7.8 \text { years (average): } \\
59 \% \text { no relapse }\end{array}$ & - \\
\hline $\begin{array}{l}\text { 1999, Paris \& } \\
\text { Canavan (2) [29] }\end{array}$ & $\begin{array}{l}\text { Physician } \\
\text { Health } \\
\text { Program }\end{array}$ & - & $\begin{array}{l}\text { after treatment } \\
\text { completion }\end{array}$ & urine & - & $\begin{array}{l}\text { participate in an aftercare group } \\
\text { for } 1 \text { year, monthly face-to-face } \\
\text { appointment with PHP employee, and } \\
\text { attendance of Alcoholics } \\
\text { Anonymous meetings }\end{array}$ & $\begin{array}{l}7.2 \text { years (average): } \\
56 \% \text { no relapse }\end{array}$ & - \\
\hline 2004, Warhaft [30] & $\begin{array}{l}\text { Case } \\
\text { Management, } \\
\text { Aftercare and } \\
\text { Monitoring } \\
\text { Program }\end{array}$ & - & $\begin{array}{l}\text { after treatment } \\
\text { completion }\end{array}$ & $\begin{array}{l}\text { urine and/or } \\
\text { breath }\end{array}$ & yes & $\begin{array}{l}\text { attendance at Caduceus group } \\
\text { andattendance at mutual help group } \\
\text { (Alcoholics or Narcotics Anonymous) }\end{array}$ & $\begin{array}{l}0 \text { to } 3 \text { years: } \\
79 \% \text { no relapse }\end{array}$ & $\begin{array}{l}0 \text { to } 3 \text { years: } \\
78 \% \text { working }\end{array}$ \\
\hline $\begin{array}{l}\text { 2005, Domino et al. } \\
\text { (1) [31] }\end{array}$ & $\begin{array}{l}\text { Washington } \\
\text { Physician } \\
\text { Health } \\
\text { Program }\end{array}$ & - & $\begin{array}{l}\text { after treatment } \\
\text { completion }\end{array}$ & urine & yes & $\begin{array}{l}\text { frequent contact for behavioral } \\
\text { assessment and regulatory board reports }\end{array}$ & $\begin{array}{l}0 \text { to } 5 \text { years: } \\
78 \% \text { no relapse }\end{array}$ & - \\
\hline $\begin{array}{l}\text { 2005, Domino et al. } \\
\text { (2) [31] }\end{array}$ & $\begin{array}{l}\text { Washington } \\
\text { Physician } \\
\text { Health } \\
\text { Program }\end{array}$ & - & $\begin{array}{l}\text { after treatment } \\
\text { completion }\end{array}$ & urine & yes & $\begin{array}{l}\text { frequent contact for behavioral } \\
\text { assessment and regulatory board reports }\end{array}$ & $\begin{array}{l}\text { more than } 5 \text { years: } \\
68 \% \text { no relapse }\end{array}$ & $\begin{array}{l}\text { more than } \\
5 \text { years: } \\
88 \% \text { working }\end{array}$ \\
\hline
\end{tabular}


Table 3. Cont.

\begin{tabular}{|c|c|c|c|c|c|c|c|c|}
\hline \multirow[b]{2}{*}{ Year, Study } & \multirow{2}{*}{$\begin{array}{l}\text { Name of the } \\
\text { Health } \\
\text { Program }\end{array}$} & \multirow[b]{2}{*}{$\begin{array}{l}\text { Recommended } \\
\text { Type of } \\
\text { Treatment }\end{array}$} & \multicolumn{4}{|c|}{ Monitoring Elements } & \multicolumn{2}{|c|}{ Monitoring Outcomes } \\
\hline & & & $\begin{array}{l}\text { Start of } \\
\text { Monitoring }\end{array}$ & $\begin{array}{l}\text { Biological } \\
\text { Monitoring }\end{array}$ & $\begin{array}{l}\text { Monitoring at } \\
\text { Work }\end{array}$ & Other Agreements & $\begin{array}{l}\text { Follow-Up: } \\
\% \text { Abstinence }\end{array}$ & $\begin{array}{l}\text { Follow-Up: } \\
\text { \% Work } \\
\text { Retention }\end{array}$ \\
\hline $\begin{array}{l}\text { 2005, Ganley et al. } \\
\text { (1) [32] }\end{array}$ & $\begin{array}{l}\text { North Carolina } \\
\text { Physician } \\
\text { Health } \\
\text { Program }\end{array}$ & $\begin{array}{l}\text { inpatient } \\
\text { and/or } \\
\text { outpatient }\end{array}$ & - & $\begin{array}{l}\text { urine } \\
\text { hair }\end{array}$ & & $\begin{array}{l}\text { meetings with volunteer monitor, } \\
\text { participate in Alcoholics } \\
\text { Anonymous and other self-help groups, } \\
\text { and participate in Caduceus meetings }\end{array}$ & $\begin{array}{l}1 \text { to } 6 \text { years: } \\
65 \% \text { no relapse } \\
26 \% \text { brief relapse }\end{array}$ & - \\
\hline $\begin{array}{l}\text { 2006, Clark } \\
\text { et al. [33] }\end{array}$ & $\begin{array}{l}\text { Program for } \\
\text { Recovering } \\
\text { Nurses }\end{array}$ & $\begin{array}{l}\text { mainly } \\
\text { outpatient } \\
(69 \%)\end{array}$ & $\begin{array}{l}\text { with treatment } \\
\text { initiation }\end{array}$ & urine & yes & $\begin{array}{l}\text { aftercare counseling and attendance at } \\
\text { recovery nursing support groups }\end{array}$ & $\begin{array}{l}3.8 \text { years (average): } \\
48 \% \text { no relapse }\end{array}$ & $\begin{array}{l}3.8 \text { years } \\
\text { (average): } \\
43 \% \text { working }\end{array}$ \\
\hline $\begin{array}{l}\text { 2007, Galanter } \\
\text { et al. [34] }\end{array}$ & $\begin{array}{l}\text { Committee for } \\
\text { Physician } \\
\text { Health }\end{array}$ & $\begin{array}{l}\text { inpatient } \\
\text { and/or } \\
\text { outpatient }\end{array}$ & $\begin{array}{l}\text { with treatment } \\
\text { initiation }\end{array}$ & urine & yes & 12-step/therapy monitor & $\begin{array}{l}3.4 \text { years (average): } \\
63 \% \text { no relapse }\end{array}$ & - \\
\hline $\begin{array}{l}\text { 2007, Knight } \\
\text { et al. [13] }\end{array}$ & $\begin{array}{l}\text { Physician } \\
\text { Health } \\
\text { Services }\end{array}$ & $\begin{array}{l}\text { individual } \\
\text { psychotherapy }\end{array}$ & $\begin{array}{l}\text { with treatment } \\
\text { initiation }\end{array}$ & urine & yes & $\begin{array}{l}\text { attendance at Caduceus meetings } \\
\text { or Alcoholics Anonymous }\end{array}$ & $\begin{array}{l}0 \text { to } 3 \text { years: } \\
56 \% \text { no relapse }\end{array}$ & - \\
\hline $\begin{array}{l}\text { 2008, Brewster } \\
\text { et al. [35] }\end{array}$ & $\begin{array}{l}\text { Ontario } \\
\text { Physician } \\
\text { Health } \\
\text { Program }\end{array}$ & $\begin{array}{l}\text { usually } \\
\text { inpatient } \\
\text { abstinence } \\
\text { based } \\
4-6 \text { weeks }\end{array}$ & $\begin{array}{l}\text { after treatment } \\
\text { completion }\end{array}$ & urine & yes & $\begin{array}{l}\text { visits to addiction medicine doctor, visits } \\
\text { to a family doctor for routine health } \\
\text { needs, and attendance at mutual support } \\
\text { groups in community }\end{array}$ & $\begin{array}{l}5 \text { years (exact): } \\
71 \% \text { no relapse } \\
\text { and } \\
14 \% \text { brief relapse }\end{array}$ & - \\
\hline $\begin{array}{l}\text { 2009, Du Pont } \\
\text { et al. [9] }\end{array}$ & $\begin{array}{l}16 \text { American } \\
\text { Physician } \\
\text { Health } \\
\text { Programs }\end{array}$ & $\begin{array}{l}\text { inpatient } \\
\text { and/or } \\
\text { outpatient }\end{array}$ & $\begin{array}{l}\text { after treatment } \\
\text { completion }\end{array}$ & urine & - & $\begin{array}{l}\text { participate in Alcoholics or } \\
\text { Narcotics Anonymous groups, } \\
\text { participate in aftercare groups, and } \\
\text { follow-up from Physician Health } \\
\text { Program monitor }\end{array}$ & $\begin{array}{l}4.5 \text { years (average): } \\
78 \% \text { no relapse }\end{array}$ & $\begin{array}{l}4.5 \text { years } \\
\text { (average): } \\
72 \% \text { working }\end{array}$ \\
\hline
\end{tabular}


Table 3. Cont.

\begin{tabular}{|c|c|c|c|c|c|c|c|c|}
\hline \multirow[b]{2}{*}{ Year, Study } & \multirow{2}{*}{$\begin{array}{l}\text { Name of the } \\
\text { Health } \\
\text { Program }\end{array}$} & \multirow{2}{*}{$\begin{array}{l}\text { Recommended } \\
\text { Type of } \\
\text { Treatment }\end{array}$} & \multicolumn{4}{|c|}{ Monitoring Elements } & \multicolumn{2}{|c|}{ Monitoring Outcomes } \\
\hline & & & $\begin{array}{l}\text { Start of } \\
\text { Monitoring }\end{array}$ & $\begin{array}{l}\text { Biological } \\
\text { Monitoring }\end{array}$ & $\begin{array}{l}\text { Monitoring at } \\
\text { Work }\end{array}$ & Other Agreements & $\begin{array}{l}\text { Follow-Up: } \\
\% \text { Abstinence }\end{array}$ & $\begin{array}{l}\text { Follow-Up: } \\
\% \text { Work } \\
\text { Retention }\end{array}$ \\
\hline $\begin{array}{l}\text { 2009, Fogger \& } \\
\text { McGuinness } \\
\text { (1) [36] }\end{array}$ & $\begin{array}{l}\text { Voluntary } \\
\text { Discipline } \\
\text { Alternative } \\
\text { Program }\end{array}$ & $\begin{array}{l}\text { inpatient } \\
\text { and/or } \\
\text { outpatient }\end{array}$ & $\begin{array}{l}\text { after treatment } \\
\text { completion }\end{array}$ & urine & - & - & $\begin{array}{l}3 \text { years (average): } \\
94 \% \text { no relapse }\end{array}$ & $\begin{array}{l}2.5 \text { years } \\
\text { (average): } \\
90 \% \text { working }\end{array}$ \\
\hline $\begin{array}{l}\text { 2011, Merlo } \\
\text { et al. [37] }\end{array}$ & $\begin{array}{l}\text { Professional } \\
\text { Resource } \\
\text { Network }\end{array}$ & - & $\begin{array}{l}\text { after treatment } \\
\text { completion }\end{array}$ & urine & - & $\begin{array}{l}\text { pharmacotherapy with naltrexone for at } \\
\text { least } 2 \text { years }\end{array}$ & $\begin{array}{l}3.4 \text { years (average): } \\
91 \% \text { no relapse }\end{array}$ & $\begin{array}{l}3.4 \text { years } \\
\text { (average): } \\
82 \% \text { working }\end{array}$ \\
\hline $\begin{array}{l}\text { 2013, Angres } \\
\text { et al. [38] }\end{array}$ & $\begin{array}{l}\text { After-Care } \\
\text { program }\end{array}$ & $\begin{array}{l}\text { abstinence } \\
\text { based } \\
\text { 6-8 weeks }\end{array}$ & $\begin{array}{l}\text { after treatment } \\
\text { completion }\end{array}$ & urine & - & $\begin{array}{l}\text { participate in Caduceus aftercare group } \\
\text { weekly }\end{array}$ & $\begin{array}{l}2 \text { years (exact): } \\
73 \% \text { no relapse }\end{array}$ & - \\
\hline $\begin{array}{l}\text { 2013, Cross } \\
\text { et al. [39] }\end{array}$ & $\begin{array}{l}\text { Chicago } \\
\text { treatment } \\
\text { program for } \\
\text { professionals }\end{array}$ & $\begin{array}{l}\text { inpatient } \\
\text { abstinence } \\
\text { based } \\
\text { 8-10 weeks }\end{array}$ & $\begin{array}{l}\text { after treatment } \\
\text { completion }\end{array}$ & urine & - & $\begin{array}{l}\text { participate in Alcoholics or } \\
\text { Narcotics Anonymous groups, } \\
\text { participate in Caduceus aftercare group, } \\
\text { and follow-up from Physician Health } \\
\text { Program monitor }\end{array}$ & $\begin{array}{l}2 \text { years (exact): } \\
87 \% \text { no relapse }\end{array}$ & - \\
\hline $\begin{array}{l}\text { 2017, Earley } \\
\text { et al. [40] }\end{array}$ & $\begin{array}{l}\text { Injectable } \\
\text { extended- } \\
\text { release } \\
\text { naltrexone }\end{array}$ & $\begin{array}{l}\text { intensive } \\
\text { outpatient }\end{array}$ & $\begin{array}{l}\text { with treatment } \\
\text { initiation }\end{array}$ & urine & - & attendance at mutual support meetings & $\begin{array}{l}2 \text { years (exact): } \\
89 \% \text { no relapse }\end{array}$ & $\begin{array}{l}2 \text { years (exact): } \\
63 \% \text { working }\end{array}$ \\
\hline $\begin{array}{l}\text { 2020, Bruguera } \\
\text { et al. [41] }\end{array}$ & $\begin{array}{l}\text { Galatea } \\
\text { Addiction } \\
\text { Programme }\end{array}$ & $\begin{array}{l}\text { inpatient }(62 \%) \\
\text { and/or } \\
\text { outpatient } \\
7-8 \text { weeks }\end{array}$ & $\begin{array}{l}\text { with treatment } \\
\text { initiation }\end{array}$ & $\begin{array}{l}\text { urine } \\
\text { hair }\end{array}$ & yes & $\begin{array}{l}\text { participate in psychotherapy } \\
\text { group weekly }\end{array}$ & $\begin{array}{l}2 \text { years (average): } \\
30 \% \text { no relapse }\end{array}$ & - \\
\hline
\end{tabular}


About half of the studies mainly included male subjects (14 studies) and physicians only (20 studies). The most commonly reported substances of abuse were opioids (22 studies) and alcohol (16 studies). Studies that indicated the source of referral to monitoring reported licensing boards (8 studies), employers (5 studies), colleagues (7 studies), family members (6 studies), treatment providers ( 2 studies), and self-referrals ( 9 studies). All health programs offered biological monitoring, whether or not in combination with monitoring at work (10 studies), and / or other monitoring arrangements (28 studies). Monitoring started either simultaneously with treatment initiation (8 studies) or after treatment completion (17 studies). Four studies did not indicate when monitoring started. Sample size varied widely between 11 to 904 healthcare professionals, with data available for a total of 3027 healthcare professionals for abstinence, and 1728 for work retention. Follow-up range also varied widely between 0 to 8 years; including 5 studies on abstinence and 2 studies on work retention with a follow-up of 5 years or more. The quality index of the included studies ranged from 0.2 to 0.9 , see Table A1.

\subsection{Abstinence}

Abstinence rates in the individual studies ranged from 30 to $94 \%$ with a substantial heterogeneity across studies $(Q=312.1 ; p<0.001 ; \mathrm{I} 2=92 \%)$. The overall pooled abstinence rate across studies was $72 \%(95 \%-C I=63-80 \%)$, with a follow-up duration up to 8 years, see Figure 2. When stratified by starting moment of monitoring, the subgroup analysis slightly reduced heterogeneity across studies and showed a higher abstinence rate among studies that started monitoring after treatment completion $(79 \% ; 95 \%-C I=72-85 \% ; Q=74.0$; $p<0.001 ; \mathrm{I} 2=80 \%$ ), compared to studies that started monitoring at treatment initiation (53\%; 95\%-CI = 40-67\%; Q = 60.3; $p<0.001 ; \mathrm{I} 2=88 \%$ ).

Subgroup analyses on the type of monitoring did slightly reduce heterogeneity across studies (Figure A1). Heterogeneity across studies was not significantly reduced by duration of follow-up, gender, type of healthcare professional, and type of substance use (Figures A2-A5). Risk of bias across studies was visualized in a Doi plot, indicating an asymmetric shape for the pooled abstinence rate (Figure A6). The LFK index was -1.59, also indicating minor publication bias.

\subsection{Work Retention}

Work retention rates of the individual studies ranged from 43 to $96 \%$ with a substantial heterogeneity across studies $(Q=162.7 ; p<0.001 ; \mathrm{I} 2=92 \%)$. The overall pooled work retention rate was $77 \%(95 \%-\mathrm{CI}=61-90 \%)$, with a follow-up duration up to 8 years (Figure 3).

Subgroup analyses on type of monitoring and type of substance use did slightly reduce heterogeneity across studies (Figures A7 and A11). Subgroup analyses on starting moment of monitoring, duration of follow-up, gender, and type of healthcare professional did not significantly reduce heterogeneity across studies (Figures 3 and A8-A10). Risk of bias across studies was visualized in a Doi plot, indicating an asymmetric shape for the pooled work retention rate (Figure A12). The LFK index was -2.70, also indicating major publication bias. 


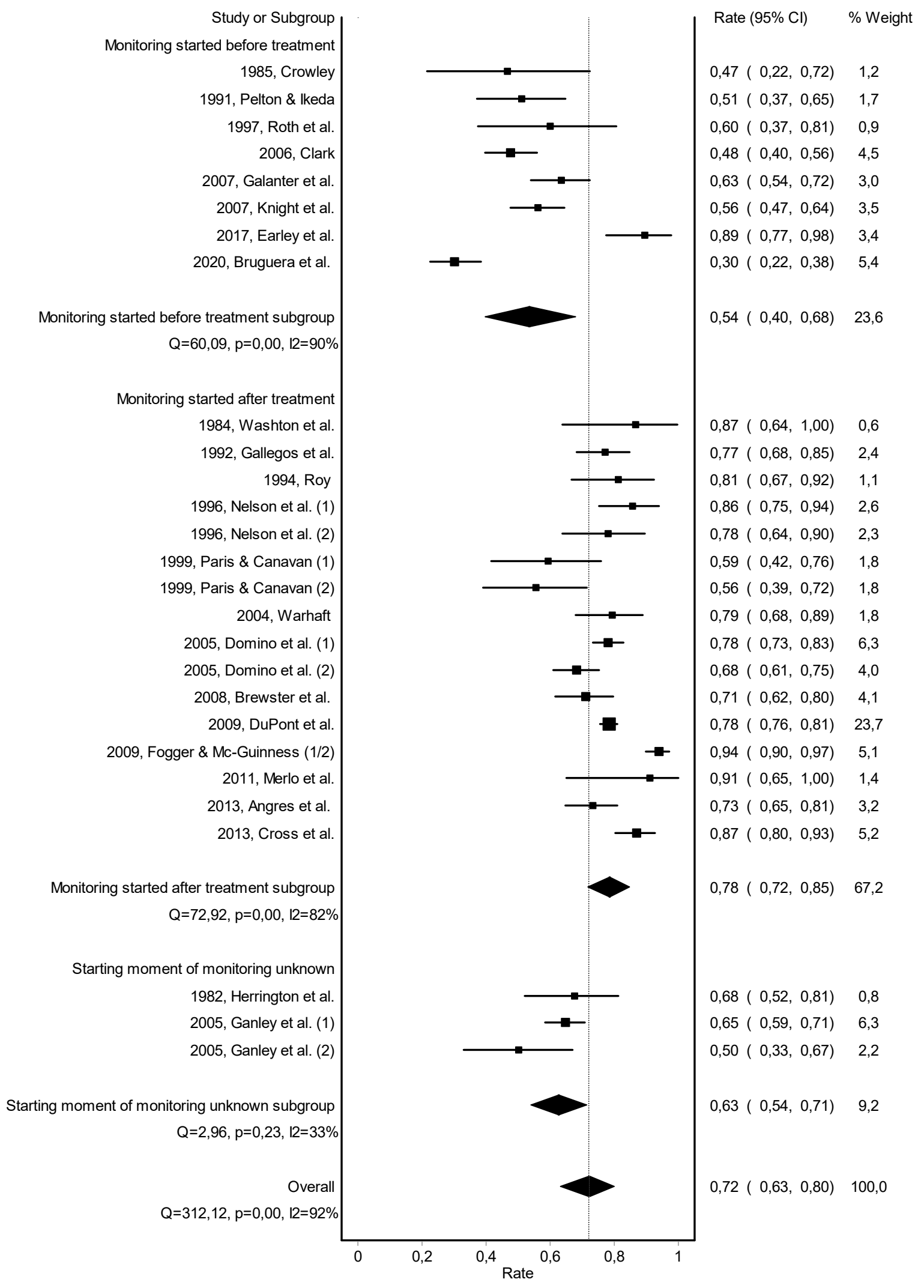

Figure 2. Forest plot of the pooled abstinence rate—subgroup analysis based on starting moment of monitoring. 


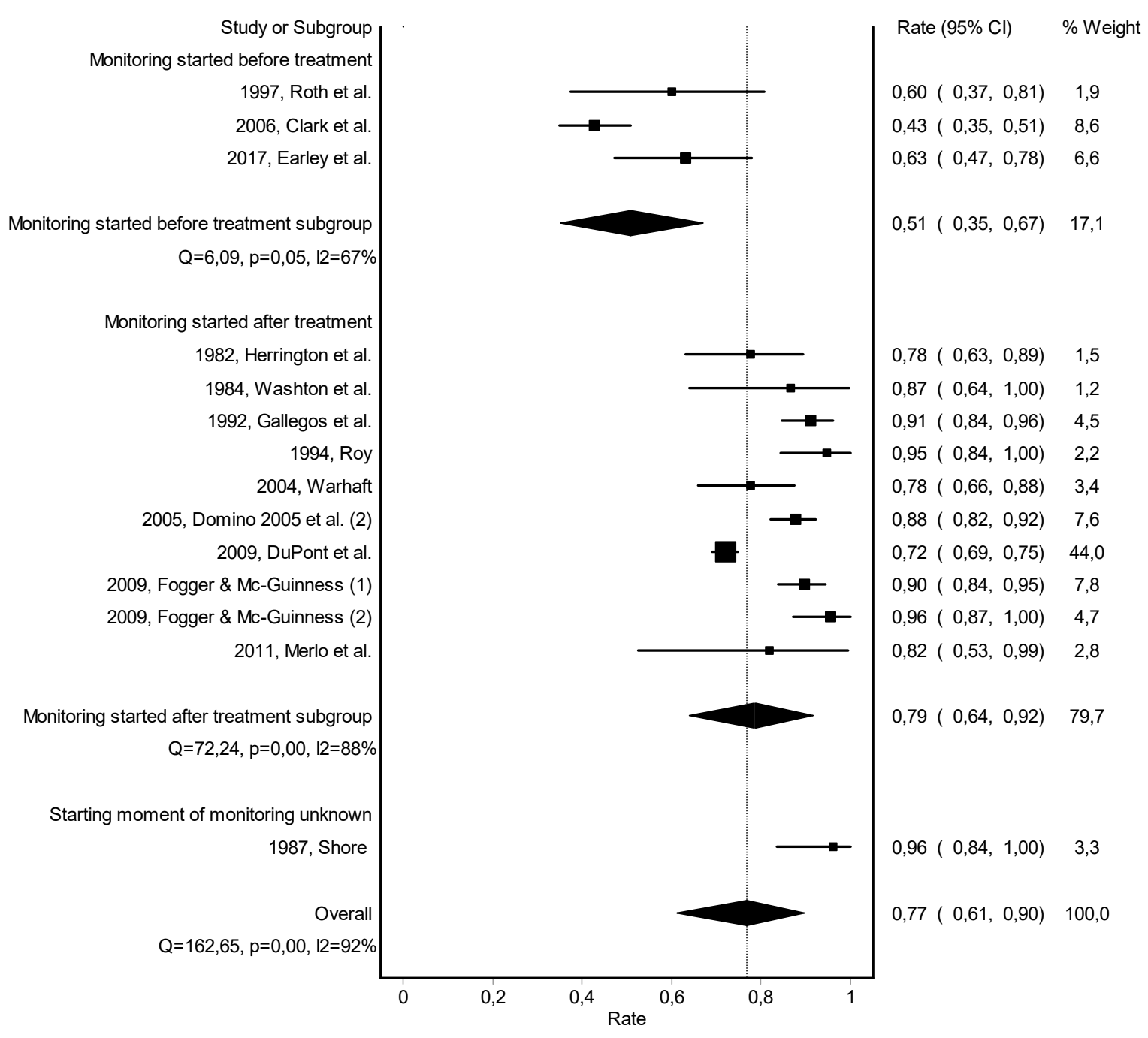

Figure 3. Forest plot of the pooled work retention rate-subgroup analysis based on starting moment of monitoring.

\section{Discussion}

This study aimed to identify the success rate of monitoring for healthcare professionals with SUD, as indexed by abstinence and work retention. Furthermore, possible explaining variables for heterogeneity were explored. On average, three quarters of the healthcare professionals who engaged in a monitoring program remained abstinent and were working at follow-up. Follow-up duration varied widely between 0 to 8 years. We identified significant heterogeneity across studies, as well as indication for publication bias. Heterogeneity within abstinence rates was partly explained by the starting moment of monitoring. Monitoring that started after successful initial treatment had better outcomes compared to those that started monitoring simultaneously with treatment. Duration of follow-up, gender, and type of healthcare professional did not significantly decrease the heterogeneity in success rates.

Unfortunately, none of the included studies used a randomized control trial or quasiexperimental design, and due to the naturalistic design of the studies included in this meta-analysis we cannot draw firm conclusions on the effectiveness of monitoring programs for healthcare professionals with SUD. If the actual effectiveness of monitoring turns out to be comparable to the success rates we found, this would be promising. In general, SUD patients show relapse rates over $50 \%$ within the first year after treatment initiation, and they remain at increased risk for relapse throughout the early years of recovery [42-44]. 
Professionals in monitoring were thus about 1.5 times more successful in maintaining abstinence when compared to regular addiction care patients without monitoring. Biological monitoring has also been applied in general SUD patients, showing a one-year abstinence rate of $46 \%$ [45,46]. This is far less successful as observed here among healthcare professionals. This may be partly attributed to the starting moment of monitoring (during treatment), but might also be the result of a difference in effectiveness of the intervention. Furthermore, work retention is a major incentive for healthcare professionals, which might apply to a lesser extent in general SUD patients. Indeed, studies on Contingency Management $(\mathrm{CM})$ and Community Reinforcement Approach (CRA) indicate that positive reinforcement increases abstinence rates [47].

We only included studies that applied biological monitoring of substance use. Biological testing is the most reliable and objective measure for abstinence [11]. The studies included in this meta-analysis mostly reported urine toxicology as method of biological testing. Yet, abstinence rates might be inflated due to false-negative urine toxicology [48] On the other hand, biological testing might be more effective in promoting abstinence than self-report. Indeed, studies on monitoring without biological testing among healthcare professionals showed somewhat less positive results (i.e., abstinence rates ranging from $13 \%$ to $76 \%$ and work retention rates ranging from $36 \%$ to $89 \%$ ) [49-58]. This might indicate that monitoring programs should preferably include biological monitoring of substance use.

Heterogeneity in abstinence rates across studies was partially explained by the starting moment of monitoring. This suggests a potential source of selection bias, depending on the timing of monitoring. Participants who start monitoring after successful treatment completion might be strongly motivated to achieve abstinence and have high chances to maintain their good treatment outcome. Moreover, the group who starts monitoring simultaneously with treatment initiation also includes participants who will drop out of treatment, or relapse during treatment. This will lead to lower success rates of monitoring. Indeed, many continuing care studies limited their participants to those who had successfully completed the initial treatment phase, thus introducing selection bias [59]. Other variables included in the subgroup analyses (duration of follow-up, gender, and type of healthcare professional) did not explain a substantial part of the heterogeneity across studies. Unfortunately, the data reported in the included studies did not enable us to perform subgroup analyses on type of initial treatment (inpatient, outpatient, pharmacological intervention, etc.) and on the mandatory status of monitoring.

Several other sources of bias might affect our findings. First, it has been suggested that many physicians who are forced to participate in a PHP might not actually have a SUD [60]. Not all PHPs use diagnostic criteria to assess their participants. Indeed, more than two-thirds of the studies included in our meta-analysis did not specify the diagnostic process of SUD assessment. Secondly, some of the studies we included did not take into account participants who were lost to follow-up in calculating the overall success rate of monitoring. It is unclear how this may have influenced the outcomes. Participants may have become lost to follow-up either because they are doing well and feel they no longer need monitoring or, on the other end of the spectrum, because they have relapsed and cannot be located or do not want to reveal their condition [59]. Thirdly, the duration of follow-up varied widely within and between the included studies and durations were either presented as range, average, or exact follow-up between 0 to 8 years. A followup of 0 years meant that some participants recently started monitoring, whereas other participants in the same study were followed-up for 3 or 5 years. Fourthly, three very small studies either showed high [21,37] or low [22] success rates, thereby possibly skewing the results. Though some success rates changed slightly, the sensitivity analyses showed that the main findings still hold, indicating the robustness of findings. Lastly, our meta-analysis showed asymmetry for both abstinence and work retention, suggesting publication bias. Taken together, this raises concerns of potential overestimation of the effectiveness of monitoring in the current literature [60]. In order to reduce reporting and publication bias, 
we strongly encourage health programs to systematically assess effectiveness and publish about the outcomes of their monitoring.

The current study results should be interpreted in the light of several limitations. First, we identified a considerable amount of heterogeneity between studies, but were able to explain only a small fraction by the starting moment of monitoring. Other potential sources of heterogeneity like the severity of the SUD, the presence of comorbidity, a (family) history of SUD, the type of initial treatment (inpatient, outpatient, pharmacological and/or psychological intervention), and the status of monitoring (mandatory or voluntary) could not be analyzed since this information was generally not available across studies $[31,33,61]$. Secondly, we included only English-language research articles published in peer-reviewed journals. This might have increased bias in our study results, because we did not include foreign language studies, unpublished studies, partially published studies, and studies published in "grey" literature sources [62]. Thirdly, the definition of the abstinence outcome measure (no relapse during follow-up) was quite strict, so some abstinence rates included in the meta-analysis were lower than reported in the conclusions of the individual studies. Furthermore, the overall quality of the included studies was moderate, with $60 \%$ of the studies scoring 0.5 or lower on the Quality Index. Thus, future studies with more rigorous designs are highly needed, in order to support effectiveness of monitoring for healthcare professionals with SUD. Finally, we focused only on healthcare professionals with SUD. Therefore, we cannot say anything about behavioral addictions or other psychiatric problems among healthcare professionals. Yet, some studies investigated the success of monitoring for other psychiatric problems among healthcare professionals, showing high recovery rates ranging from 88 to $94 \%$ and work retention rates ranging from 77 to $90 \%$ [12]. The current positive findings may thus indicate good prognosis of mental health issues in general among healthcare professionals.

\section{Conclusions}

Three quarters of the healthcare professionals who engaged in monitoring for SUD remained abstinent and were working at follow-up. There was significant heterogeneity across studies, as well as an indication for major publication bias. The heterogeneity in success rates of monitoring was slightly explained by the starting moment of monitoring, with studies starting monitoring after treatment completion showing higher success rates than studies starting monitoring at treatment initiation. Given the heterogeneity across studies and indication for publication bias, no firm conclusions can be drawn about the effectiveness of monitoring for healthcare professionals with SUD. Future studies should apply controlled comparisons, using more rigorous measurements and substantially long follow-up rates.

Supplementary Materials: The following are available online at https:/ / www.mdpi.com/2077-038 3/10/2/264/s1, Table S1: PRISMA Checklist.

Author Contributions: Conceptualization, P.M.G., S.J.M.v.d.B., B.A.G.D., J.M.K., A.H.S., F.A., and A.F.A.S.; methodology, P.M.G., F.A., and A.F.A.S.; validation, P.M.G., S.J.M.v.d.B., and B.A.G.D.; formal analysis, P.M.G. and F.A.; investigation, P.M.G., S.J.M.v.d.B., and B.A.G.D.; data curation, P.M.G.; writing-original draft preparation, P.M.G.; writing—review and editing, S.J.M.v.d.B., B.A.G.D., J.M.K., H.A.d.H., C.A.J.d.J., A.H.S., F.A., and A.F.A.S.; visualization, P.M.G.; supervision, A.F.A.S., F.A., and A.H.S.; project administration, P.M.G. and A.F.A.S.; funding acquisition, A.F.A.S., C.A.J.d.J., and H.A.d.H. All authors have read and agreed to the published version of the manuscript.

Funding: This study was funded by a grant of the Dutch Ministry of Health, Welfare and Sport (grant number 328622) to the Royal Dutch Medical Association.

Institutional Review Board Statement: Not applicable.

Informed Consent Statement: Not applicable.

Data Availability Statement: Data is contained within the supplementary material (Dataset S1: Search strategy, Dataset S2: Forest plots abstinence, Dataset S3: Forest plots work retention). 
Acknowledgments: The authors wish to thank Marlies de Rond, from the Royal Dutch Medical Association for facilitating the writing and discussion process.

Conflicts of Interest: The authors declare no conflict of interest.

\section{Appendix A}

Table A1. The Health States Quality Index.

\begin{tabular}{|c|c|c|c|c|c|c|c|c|}
\hline \multirow[t]{2}{*}{ Year, Study } & \multicolumn{6}{|c|}{ Variables } & \multirow[t]{2}{*}{$\begin{array}{c}\text { Total } \\
(\mathbf{M a x}=10)\end{array}$} & \multirow[t]{2}{*}{ Quality Index } \\
\hline & 1 & 2 & 3 & 4 & 5 & 6 & & \\
\hline 1982, Herrington et al. & 1 & 0 & 1 & 0 & 0 & 0 & 2 & 0.2 \\
\hline 1984, Washton et al. & 1 & 0 & 1 & 0 & 0 & 0 & 2 & 0.2 \\
\hline 1985, Crowley & 0 & 0 & 1 & 1 & 1 & 1 & 4 & 0.4 \\
\hline 1987, Shore & 0 & 1 & 2 & 1 & 1 & 0 & 5 & 0.5 \\
\hline 1991, Pelton \& Ikeda & 0 & 0 & 2 & 1 & 1 & 0 & 4 & 0.4 \\
\hline 1992, Gallegos et al. & 1 & 0 & 1 & 1 & 1 & 0 & 4 & 0.4 \\
\hline 1994, Roy & 0 & 0 & 1 & 0 & 1 & 1 & 3 & 0.3 \\
\hline 1996, Nelson et al. (1) & 1 & 0 & 2 & 1 & 1 & 1 & 6 & 0.6 \\
\hline 1996, Nelson et al. (2) & 1 & 0 & 2 & 1 & 1 & 1 & 6 & 0.6 \\
\hline 1997, Roth et al. & 0 & 0 & 1 & 1 & 0 & 1 & 3 & 0.3 \\
\hline 1999, Paris \& Canavan (1) & 0 & 0 & 2 & 1 & 1 & 1 & 5 & 0.5 \\
\hline 1999, Paris \& Canavan (2) & 0 & 0 & 2 & 1 & 1 & 1 & 5 & 0.5 \\
\hline 2004, Warhaft & 1 & 0 & 2 & 0 & 1 & 0 & 4 & 0.4 \\
\hline 2005, Domino et al. (1) & 1 & 0 & 2 & 1 & 1 & 0 & 5 & 0.5 \\
\hline 2005, Domino et al. (2) & 1 & 0 & 2 & 1 & 1 & 0 & 5 & 0.5 \\
\hline 2005, Ganley et al. (1) & 1 & 1 & 2 & 1 & 1 & 0 & 6 & 0.6 \\
\hline 2005, Ganley et al. (2) & 1 & 1 & 2 & 1 & 1 & 0 & 6 & 0.6 \\
\hline 2006, Clark et al. & 1 & 0 & 2 & 1 & 1 & 1 & 6 & 0.6 \\
\hline 2007, Galanter et al. & 1 & 0 & 1 & 1 & 1 & 1 & 5 & 0.5 \\
\hline 2007, Knight et al. & 1 & 0 & 2 & 1 & 1 & 0 & 5 & 0.5 \\
\hline 2008, Brewster et al. & 1 & 1 & 1 & 1 & 1 & 2 & 7 & 0.7 \\
\hline 2009, DuPont et al. & 1 & 0 & 2 & 1 & 2 & 1 & 7 & 0.7 \\
\hline $\begin{array}{c}\text { 2009, Fogger \& } \\
\text { Mc-Guinness (1) }\end{array}$ & 0 & 0 & 2 & 2 & 1 & 1 & 6 & 0.6 \\
\hline $\begin{array}{c}\text { 2009, Fogger \& } \\
\text { Mc-Guinness (2) }\end{array}$ & 0 & 0 & 2 & 2 & 1 & 1 & 6 & 0.6 \\
\hline 2011, Merlo et al. & 0 & 0 & 2 & 1 & 1 & 1 & 5 & 0.5 \\
\hline 2013, Cross et al. & 1 & 1 & 2 & 1 & 1 & 2 & 8 & 0.8 \\
\hline 2013, Angres et al. & 0 & 1 & 1 & 1 & 0 & 2 & 5 & 0.5 \\
\hline 2017, Earley et al. & 1 & 1 & 1 & 2 & 2 & 2 & 9 & 0.9 \\
\hline 2020, Bruguera et al. & 1 & 1 & 2 & 1 & 2 & 1 & 8 & 0.8 \\
\hline
\end{tabular}


Table A1. Cont.

\begin{tabular}{cccc}
\hline Variables & $\mathbf{0}$ & $\mathbf{1}$ & $\mathbf{2}$ \\
\hline $\begin{array}{c}\text { 1. Clear definition of target } \\
\text { population and observation period }\end{array}$ & No & Yes & Attempts all cases \\
\hline 2. Use of diagnostic criteria & $\begin{array}{c}\text { Symptom based } \\
\text { Not specified }\end{array}$ & $\begin{array}{c}\text { Use of diagnostic system } \\
\text { reported }\end{array}$ & Administered interview \\
\hline 3. Method of case selection & Not specified & Convenience sampling & $\begin{array}{c}\text { Register } \\
\text { Case record }\end{array}$ \\
4. Type of outcome assessment & Not specified & $\begin{array}{c}\text { Small } \\
\text { (single site) }\end{array}$ & (national or multisite) \\
\hline 5. Type of prevalence measure & $\begin{array}{c}\text { Range of follow-up } \\
\text { duration }\end{array}$ & Average follow-up duration & Exact follow-up duration \\
\hline
\end{tabular}




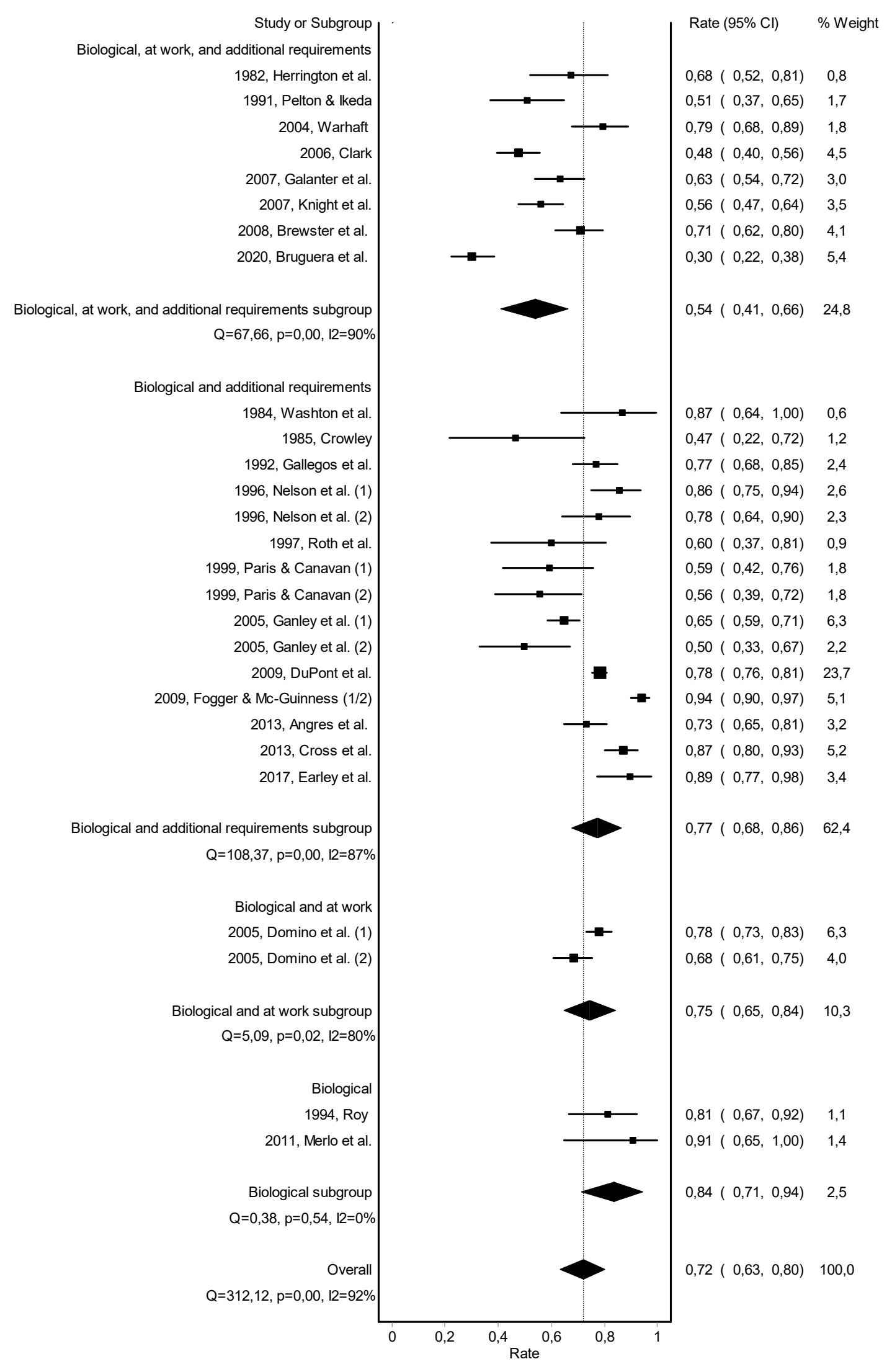

Figure A1. Forest plot of the pooled abstinence rate-subgroup analysis based on type of monitoring. 


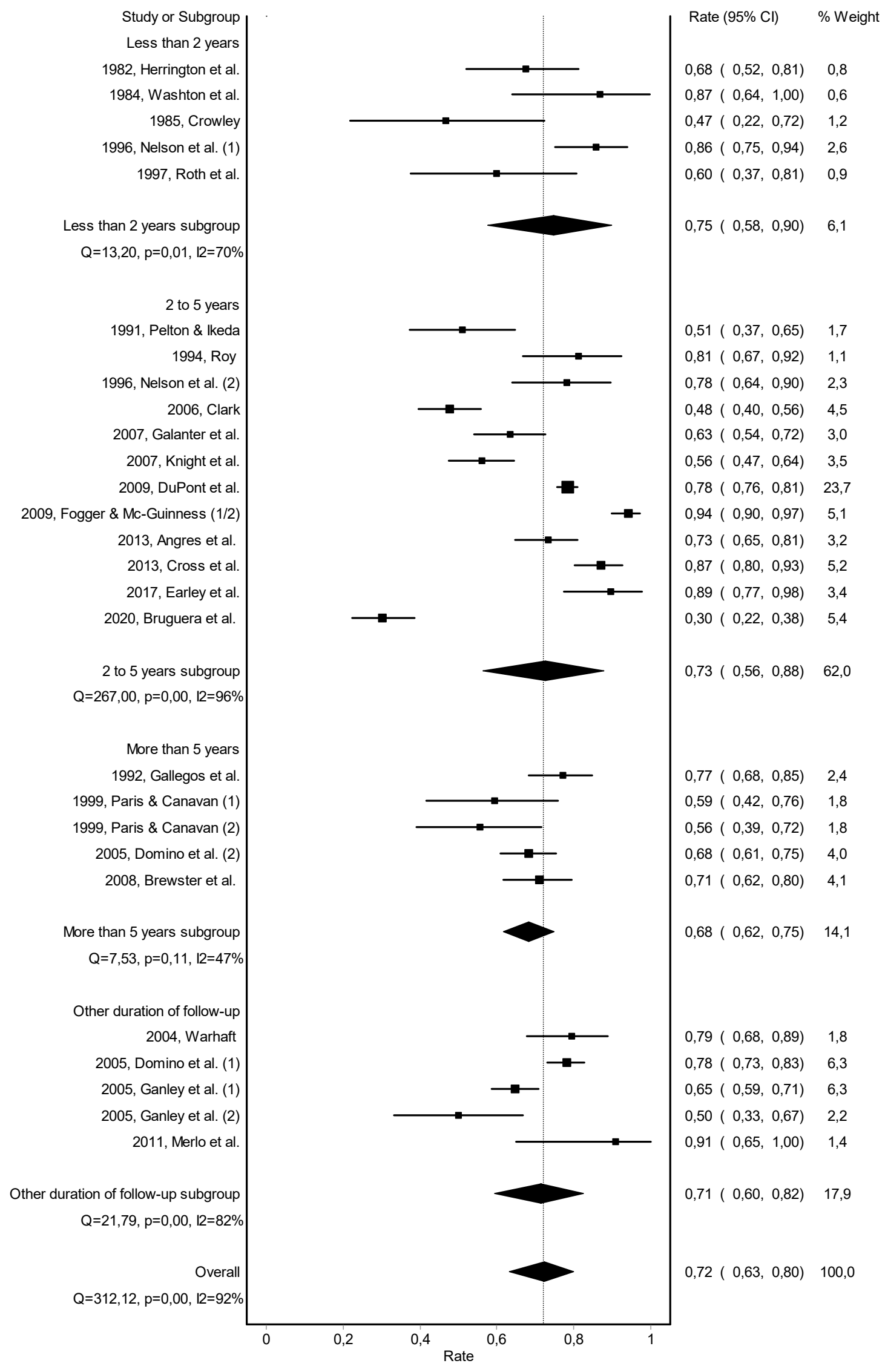

Figure A2. Forest plot of the pooled abstinence rate—subgroup analysis based on duration of follow-up. 


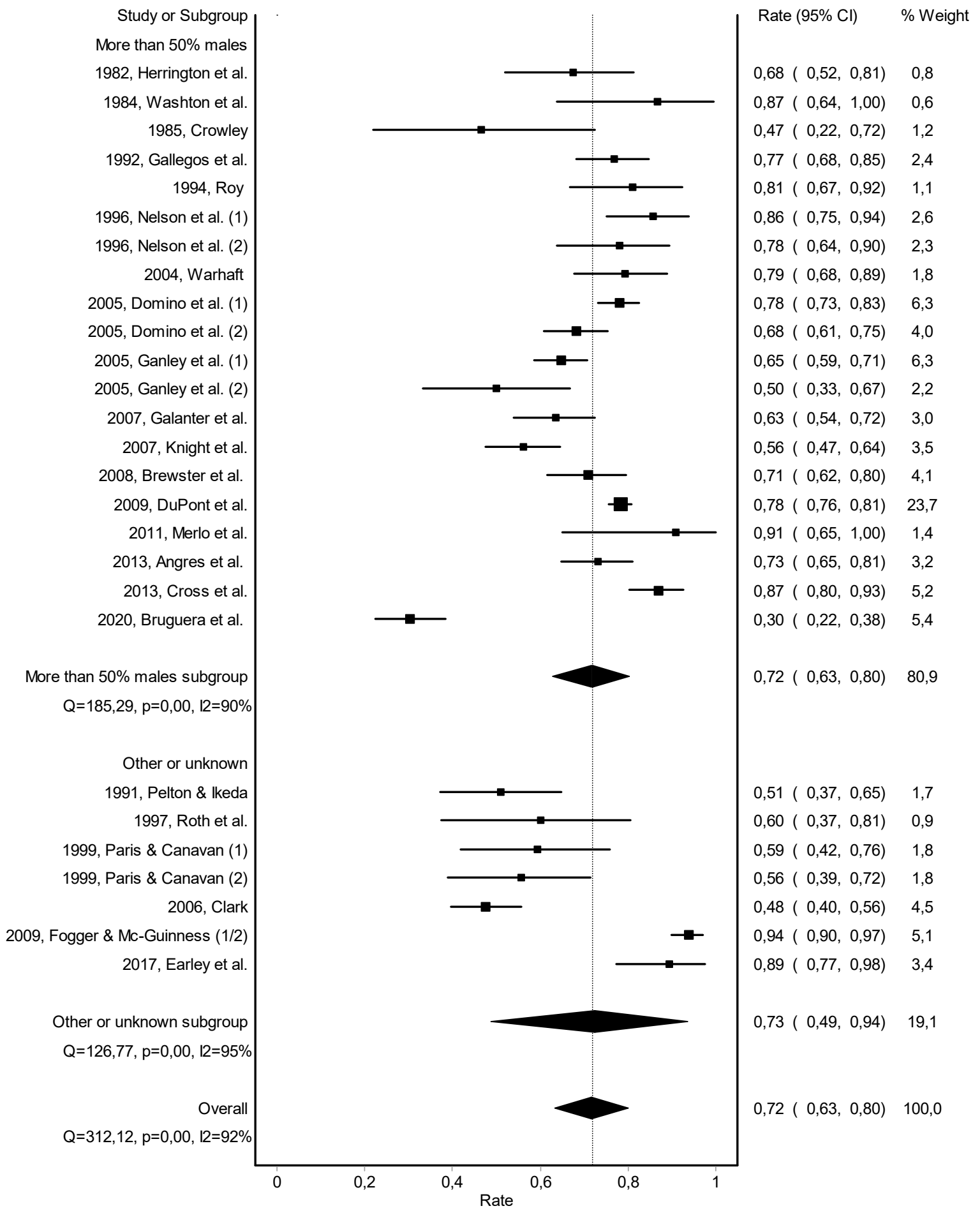

Figure A3. Forest plot of the pooled abstinence rate-subgroup analysis based on gender. 


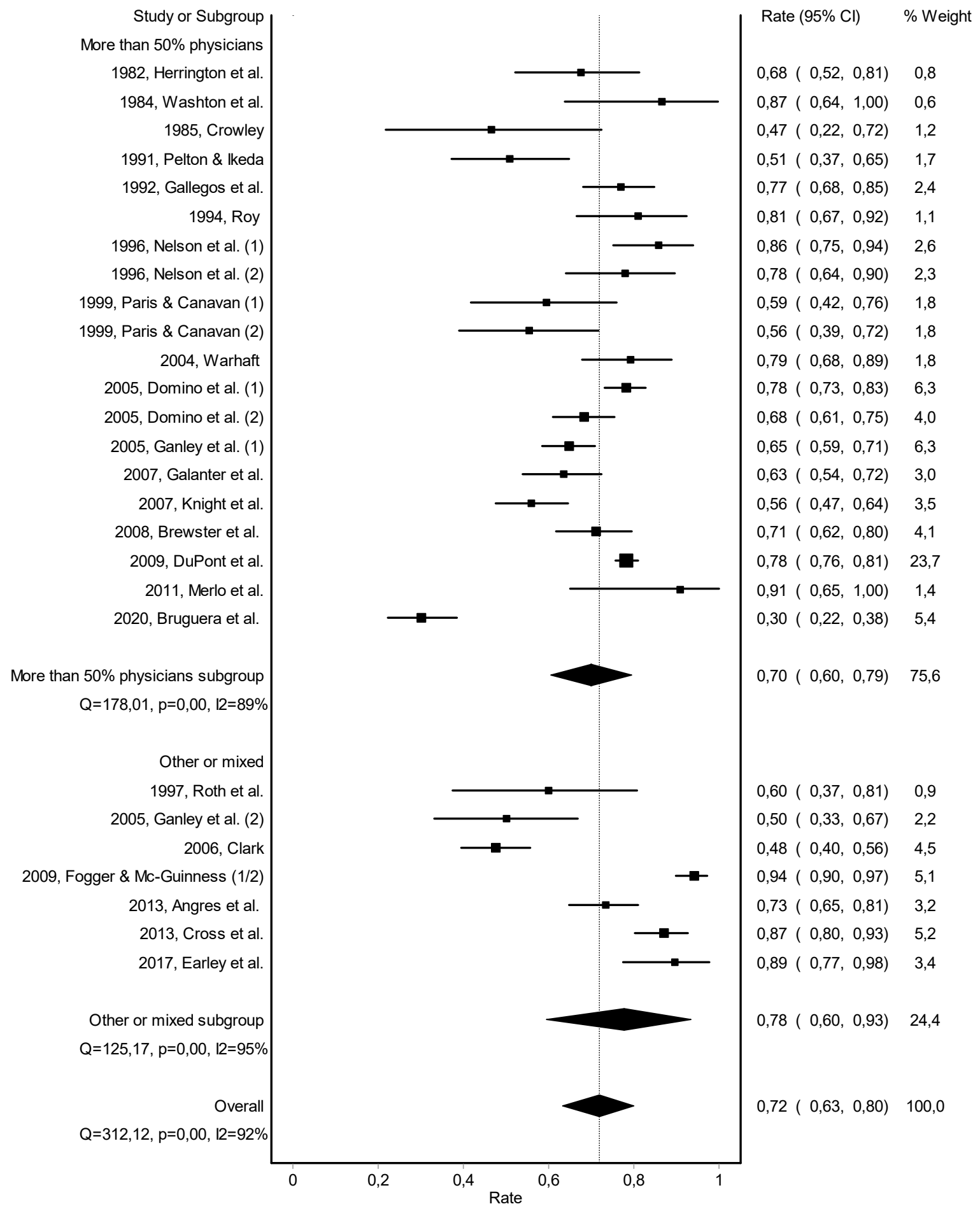

Figure A4. Forest plot of the pooled abstinence rate—subgroup analysis based on type of healthcare professional. 


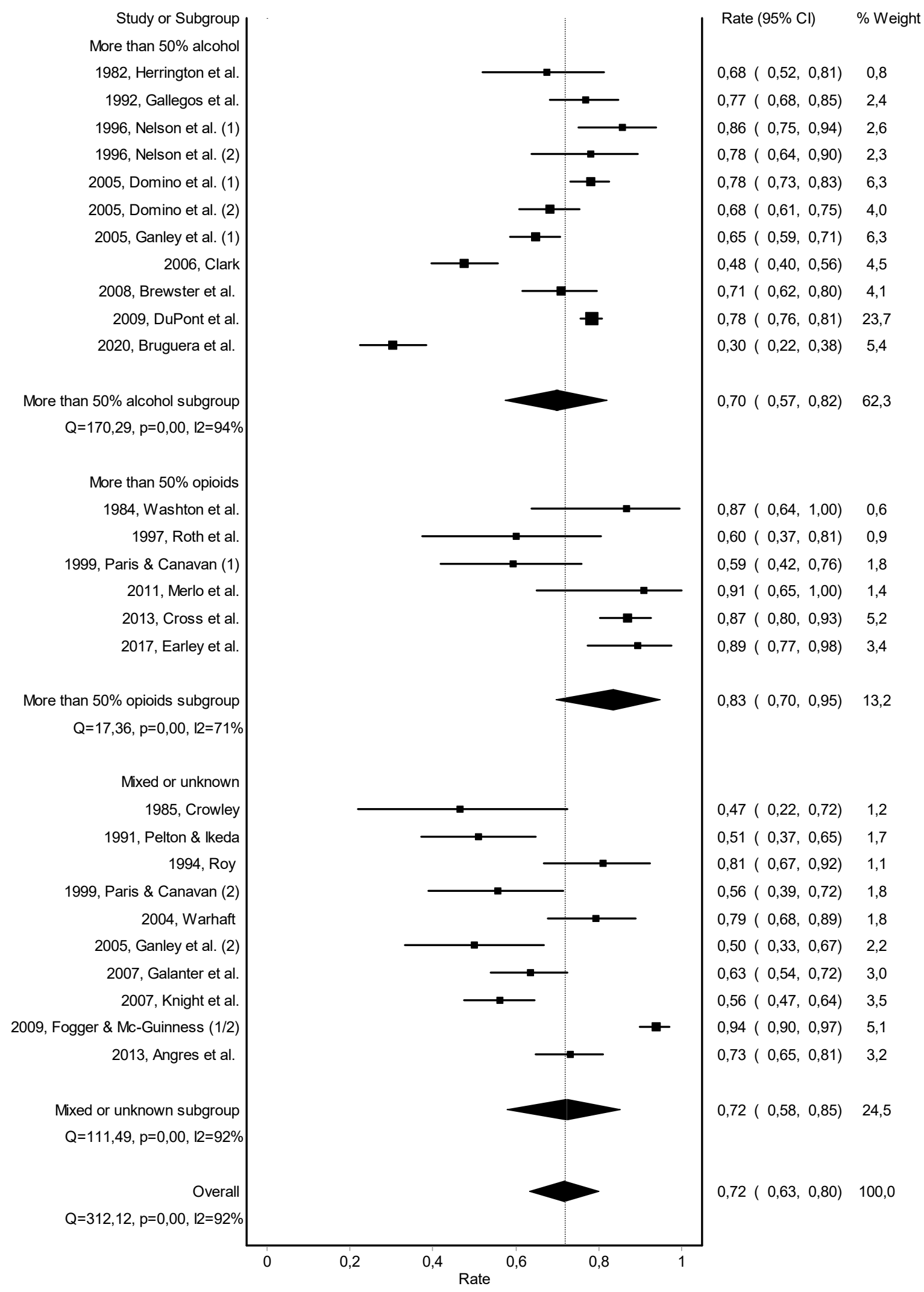

Figure A5. Forest plot of the pooled abstinence rate-subgroup analysis based on type of substance use. 


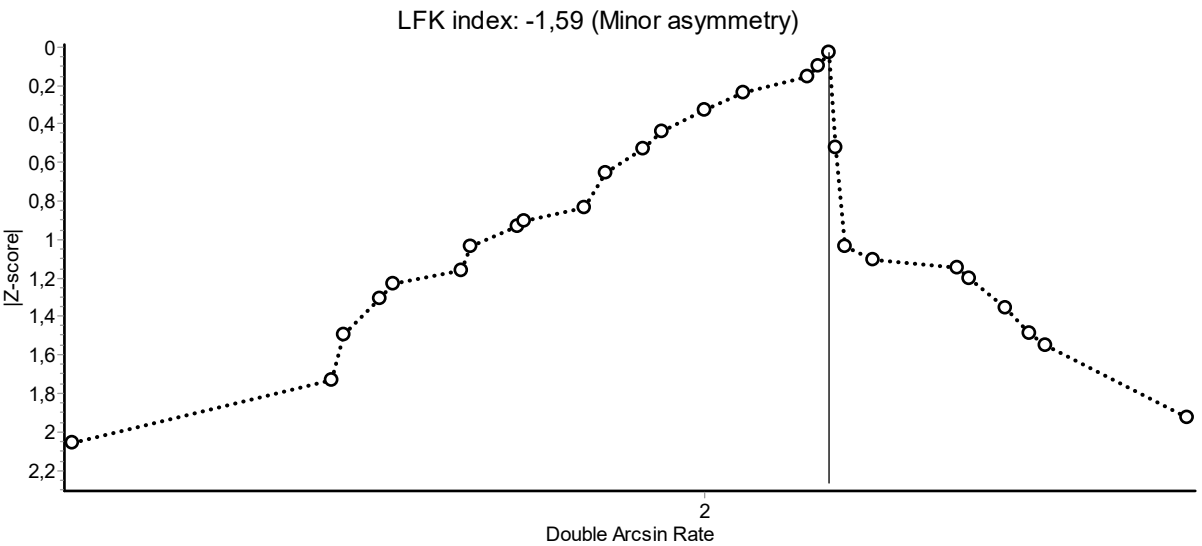

Figure A6. Doi plot analysis and Luis Furuya-Kanamori asymmetry (LFK) index of publication bias for the pooled abstinence rate.

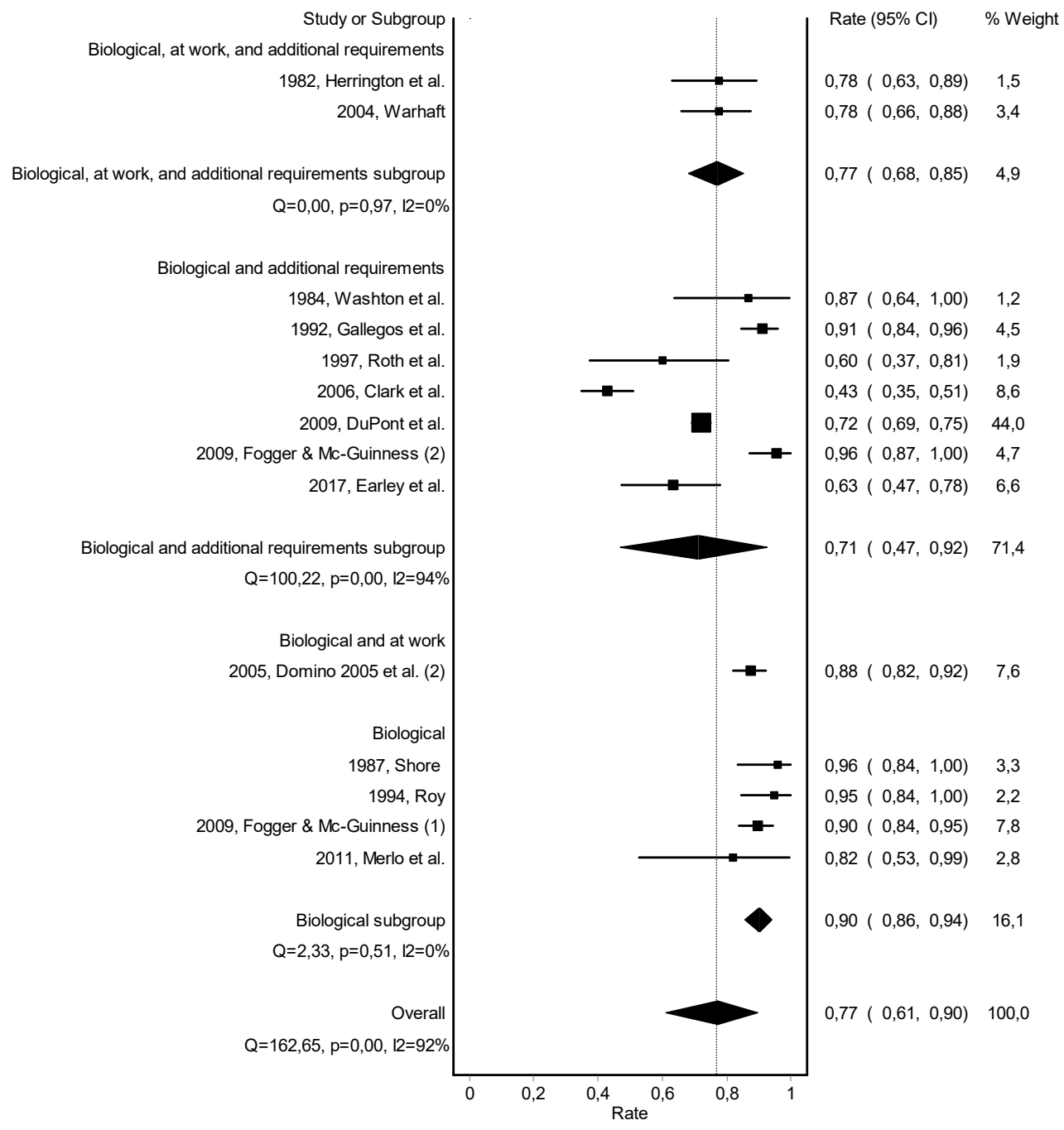

Figure A7. Forest plot of the pooled work retention rate-subgroup analysis based on type of monitoring. 


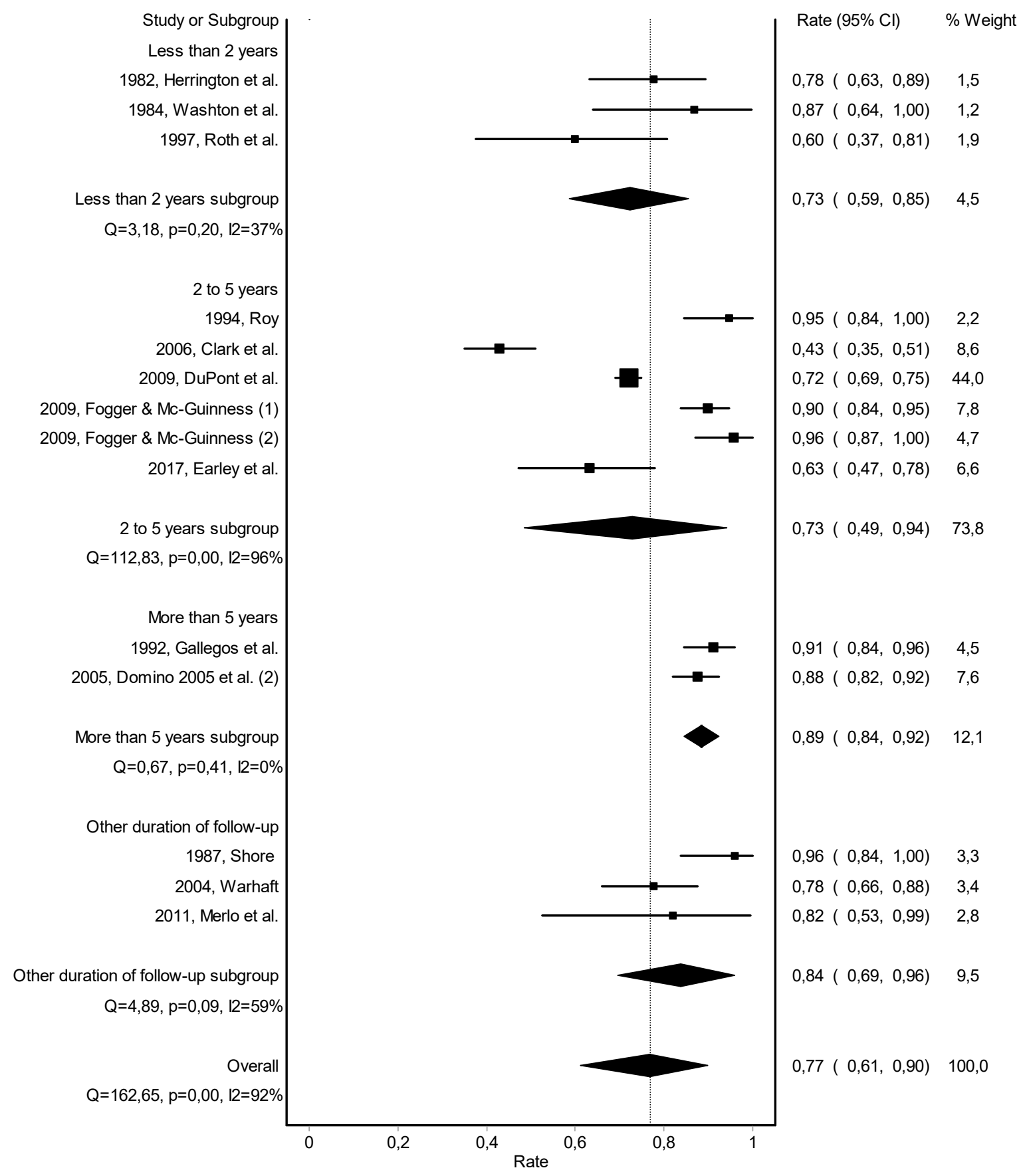

Figure A8. Forest plot of the pooled work retention rate-subgroup analysis based on duration of follow-up. 


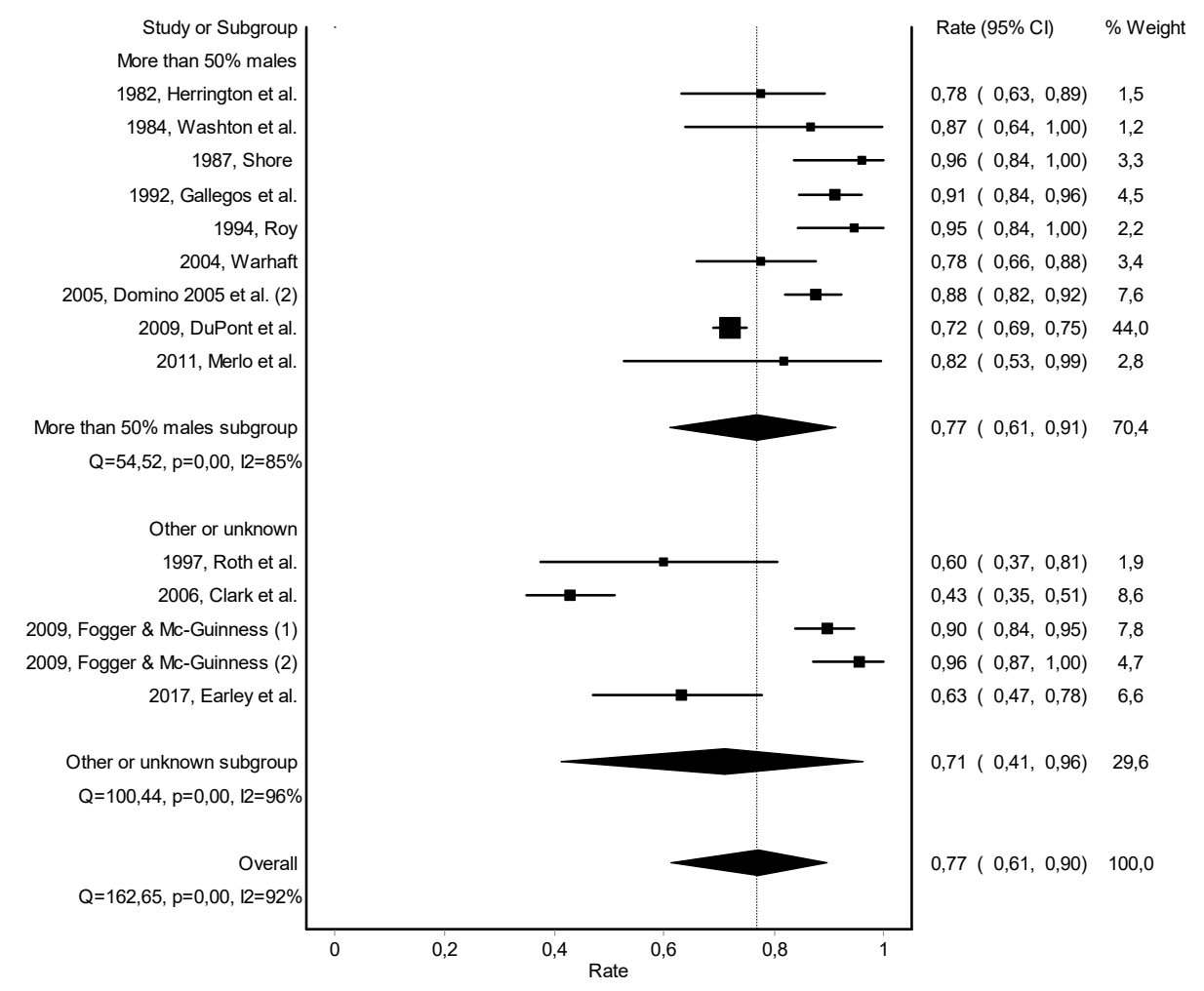

Figure A9. Forest plot of the pooled work retention rate-subgroup analysis based on gender.

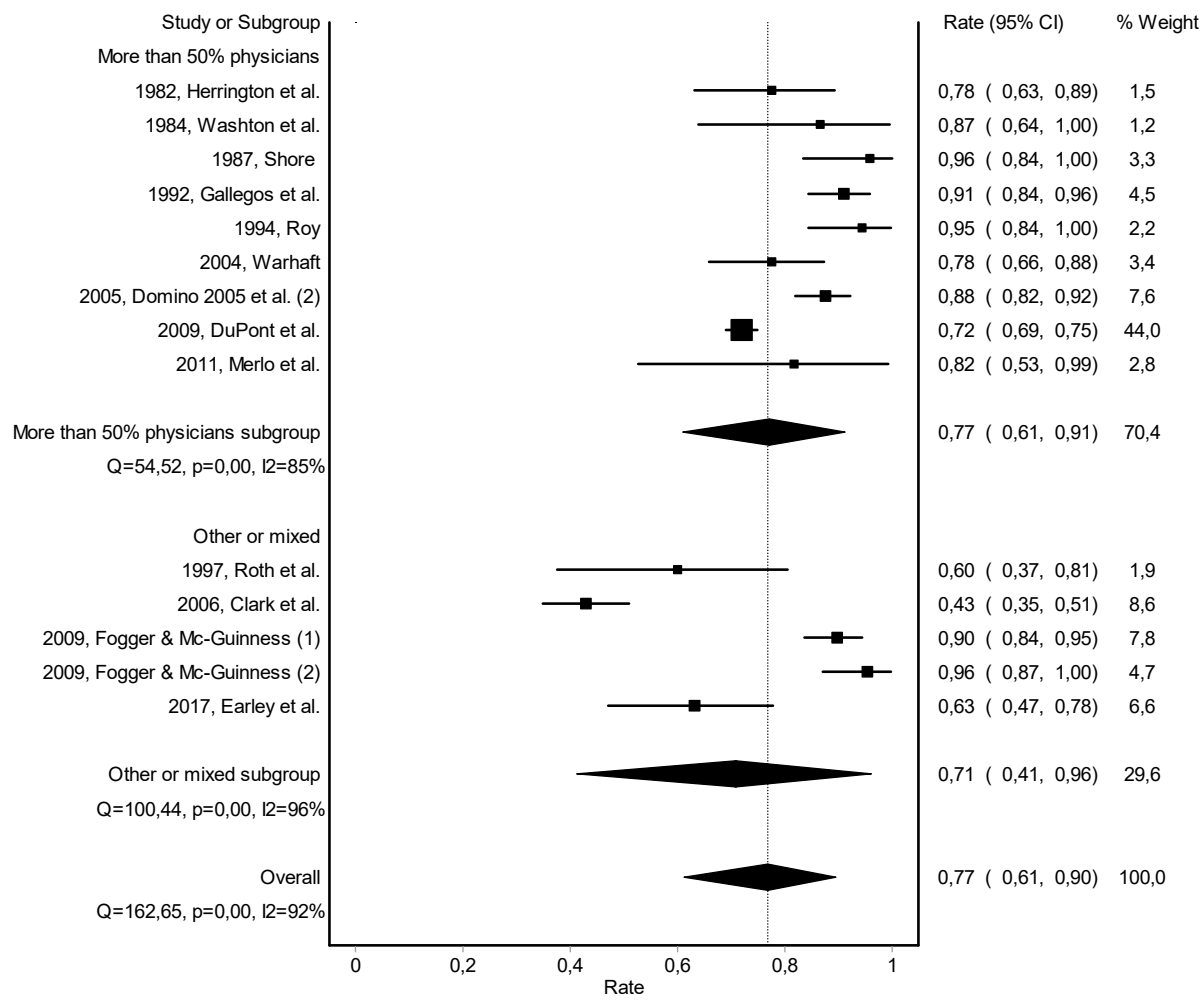

Figure A10. Forest plot of the pooled work retention rate-subgroup analysis based on type of healthcare professional. 


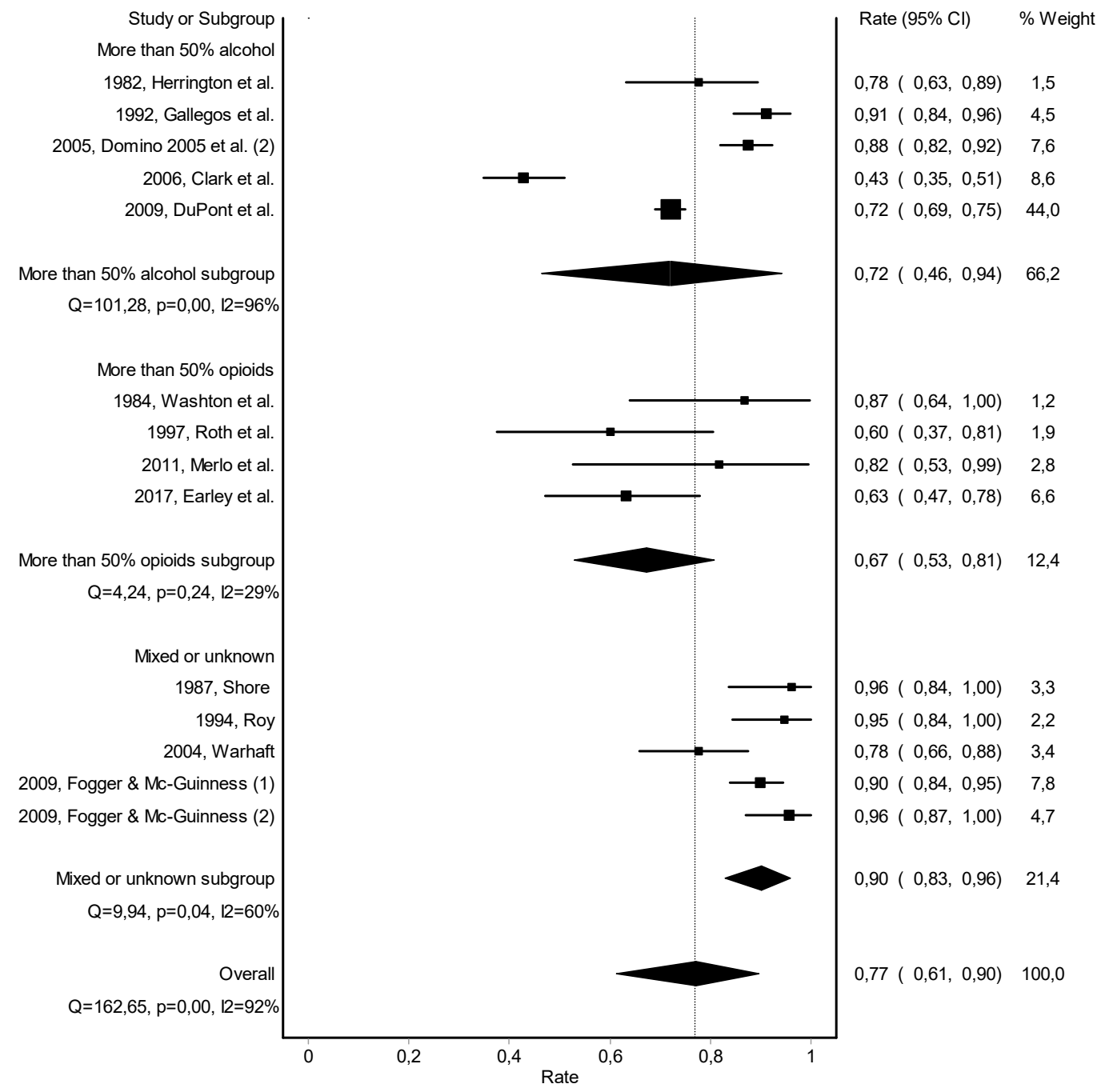

Figure A11. Forest plot of the pooled work retention rate-subgroup analysis based on type of substance use.

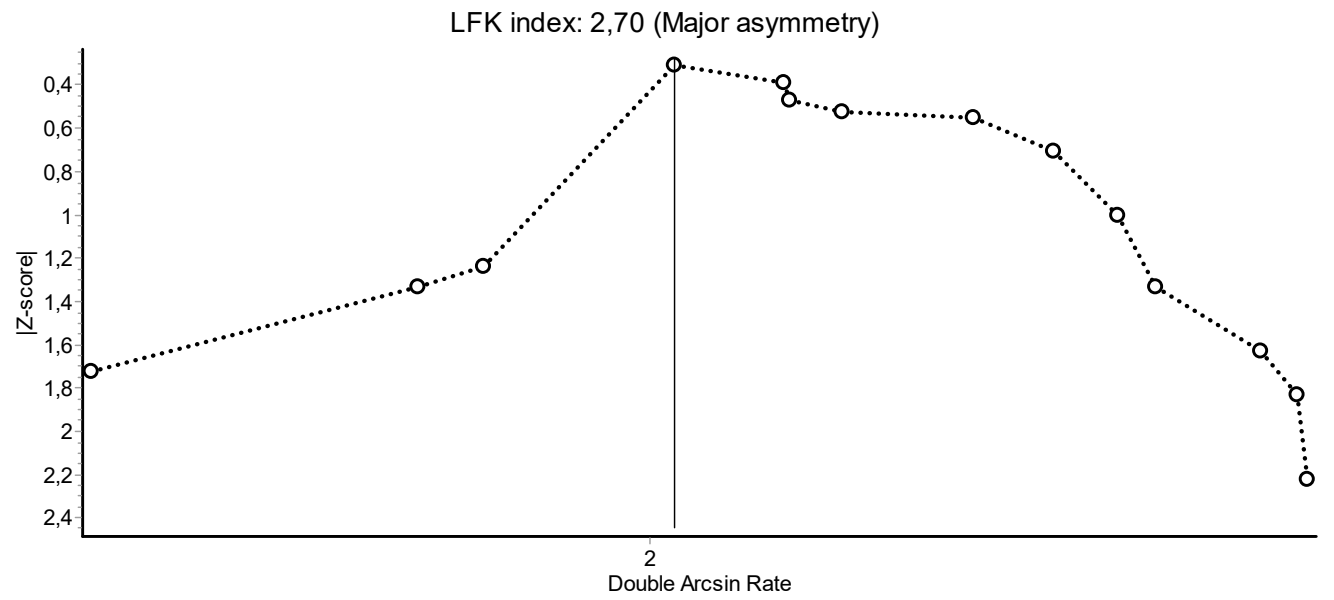

Figure A12. Doi plot analysis and Luis Furuya-Kanamori asymmetry (LFK) index of publication bias for the pooled work retention rate. 


\section{References}

1. Kunyk, D. Substance use disorders among registered nurses: Prevalence, risks and perceptions in a disciplinary jurisdiction. J. Nurs. Manag. 2015, 23, 54-64. [CrossRef] [PubMed]

2. Wallace, J.E.; Lemaire, J.B.; Ghali, W.A. Physician wellness: A missing quality indicator. Lancet 2009, 374, 1714-1721. [CrossRef]

3. Oreskovich, M.R.; Shanafelt, T.; Dyrbye, L.N.; Tan, L.; Sotile, W.; Satele, D. The prevalence of substance use disorders in American physicians. Am. J. Addict. 2015, 24, 30-38. [CrossRef]

4. Hughes, P.H.; Storr, C.; Baldwin, D.C.; Williams, K.M.; Conard, S.; Sheehan, D. Patterns of substance use in the medical profession. Med. J. 1992, 41, 311-314.

5. Trinkoff, A.M.; Eaton, W.W.; Anthony, J.C. The prevalence of substance abuse among registered nurses. Nurs. Res. 1991, 40, 172-175. [CrossRef] [PubMed]

6. American Medical Association. The sick physician. Impairment by psychiatric disorders, including alcoholism and drug dependence. JAMA 1973, 223, 684-687. [CrossRef]

7. Braquehais, M.D.; Tresidder, A.; DuPont, R.L. Service provision to physicians with mental health and addiction problems. Curr. Opin. Psychiatry 2015, 28, 324-329. [CrossRef]

8. Brooks, S.K.; Gerada, C.; Chalder, T. Review of literature on the mental health of doctors: Are specialist services needed? J. Ment. Health 2011, 20, 146-156. [CrossRef]

9. DuPont, R.L.; McLellan, A.T.; White, W.L.; Merlo, L.J.; Gold, M.S. Setting the standard for recovery: Physicians' Health Programs. J. Subst. Abus. Treat. 2009, 36, 159-171. [CrossRef]

10. Gerada, C. The Wounded Healer: Report on the First 10 Years of Practitioner Health Service; NHS: London, UK, 2018.

11. Jarvis, M.; Williams, J.; Hurford, M.; Lindsay, D.; Lincoln, P.; Giles, L. Appropriate Use of Drug Testing in Clinical Addiction Medicine. J. Addict. Med. 2017, 11, 163-173. [CrossRef]

12. Weenink, J.W.; Kool, R.B.; Bartels, R.H.; Westert, G.P. Getting back on track: A systematic review of the outcomes of remediation and rehabilitation programmes for healthcare professionals with performance concerns. BMJ Qual. Saf. 2017, 26, 1004-1014. [CrossRef] [PubMed]

13. Knight, J.R.; Sanchez, L.T.; Sherritt, L.; Bresnahan, L.R.; Fromson, J.A. Outcomes of a monitoring program for physicians with mental and behavioral health problems. J. Psychiatr. Pract. 2007, 13, 25-32. [CrossRef] [PubMed]

14. Ouzzani, M.; Hammady, H.; Fedorowicz, Z.; Elmagarmid, A. Rayyan-a web and mobile app for systematic reviews. Syst. Rev. 2016, 5, 210. [CrossRef] [PubMed]

15. Barendregt, J.; Doi, S. MetaXL User Guide; EpiGear: Sunrise Beach, Australia, 2016.

16. Moher, D.; Liberati, A.; Tetzlaff, J.; Altman, D.G.; Group, P. Preferred reporting items for systematic reviews and meta-analyses: The PRISMA statement. BMJ 2009, 339, b2535. [CrossRef]

17. Stroup, D.F.; Berlin, J.A.; Morton, S.C.; Olkin, I.; Williamson, G.D.; Rennie, D. Meta-analysis of observational studies in epidemiology: A proposal for reporting. Meta-analysis Of Observational Studies in Epidemiology (MOOSE) group. JAMA 2000, 283, 2008-2012. [CrossRef]

18. Barendregt, J.J.; Doi, S.A.; Lee, Y.Y.; Norman, R.E.; Vos, T. Meta-analysis of prevalence. J. Epidemiol. Community Health 2013, 67, 974-978. [CrossRef]

19. Doi, S.A.; Thalib, L. A quality-effects model for meta-analysis. Epidemiology 2008, 19, 94-100. [CrossRef]

20. Herrington, R.E.; Benzer, D.G.; Jacobson, G.R.; Hawkins, M.K. Treating substance-use disorders among physicians. JAMA 1982, 247, 2253-2257. [CrossRef]

21. Washton, A.M.; Gold, M.S.; Pottash, A.C. Naltrexone in addicted physicians and business executives. NIDA Res. Monogr. 1984, 55, 185-190.

22. Crowley, T.J. Doctors' drug abuse reduced during contingency-contracting treatment. Alcohol Drug Res. 1985, 6, $299-307$.

23. Shore, J.H. The Oregon experience with impaired physicians on probation. An eight-year follow-up. JAMA 1987, 257, 2931-2934. [CrossRef] [PubMed]

24. Pelton, C.; Ikeda, R.M. The California Physicians Diversion Program's experience with recovering anesthesiologists. J. Psychoact. Drugs 1991, 23, 427-431. [CrossRef] [PubMed]

25. Gallegos, K.V.; Lubin, B.H.; Bowers, C.; Blevins, J.W.; Talbott, G.D.; Wilson, P.O. Relapse and recovery: Five to ten year follow-up study of chemically dependent physicians-the Georgia experience. Md. Med. J. 1992, 41, 315-319. [PubMed]

26. Roy, A.K., 3rd. Reentry monitoring in the treatment of physicians with substance dependence. South. Med. J. 1994, 87, 881-883. [CrossRef]

27. Nelson, H.D.; Matthews, A.M.; Girard, D.E.; Bloom, J.D. Substance-impaired physicians probationary and voluntary treatment programs compared. West. J. Med. 1996, 165, 31-36.

28. Roth, A.; Hogan, I.; Farren, C. Naltrexone plus group therapy for the treatment of opiate-abusing health-care professionals. J. Subst. Abus. Treat. 1997, 14, 19-22. [CrossRef]

29. Paris, R.T.; Canavan, D.I. Physician substance abuse impairment: Anesthesiologists vs. other specialties. J. Addict. Dis. 1999, 18, 1-7. [CrossRef]

30. Warhaft, N.J. The Victorian Doctors Health Program: The first 3 years. Med. J. Aust. 2004, 181, 376-379. [CrossRef]

31. Domino, K.B.; Hornbein, T.F.; Polissar, N.L.; Renner, G.; Johnson, J.; Alberti, S. Risk factors for relapse in health care professionals with substance use disorders. JAMA 2005, 293, 1453-1460. [CrossRef] 
32. Ganley, O.H.; Pendergast, W.J.; Wilkerson, M.W.; Mattingly, D.E. Outcome study of substance impaired physicians and physician assistants under contract with North Carolina Physicians Health Program for the period 1995-2000. J. Addict. Dis. 2005, $24,1-12$. [CrossRef]

33. Clark, C.; Farnsworth, J. Program for recovering nurses: An evaluation. Medsurg Nurs. Off. J. Acad. Med.-Surg. Nurses 2006, 15, 223-230.

34. Galanter, M.; Dermatis, H.; Mansky, P.; McIntyre, J.; Perez-Fuentes, G. Substance-abusing physicians: Monitoring and twelvestep-based treatment. Am. J. Addict. 2007, 16, 117-123. [CrossRef] [PubMed]

35. Brewster, J.M.; Kaufmann, I.M.; Hutchison, S.; MacWilliam, C. Characteristics and outcomes of doctors in a substance dependence monitoring programme in Canada: Prospective descriptive study. BMJ Br. Med. J. 2008, 337, 7679. [CrossRef] [PubMed]

36. Fogger, S.A.; McGuinness, T. Alabama's nurse monitoring programs: The nurse's experience of being monitored. J. Addict. Nurs. 2009, 20, 142-149. [CrossRef]

37. Merlo, L.J.; Greene, W.M.; Pomm, R. Mandatory naltrexone treatment prevents relapse among opiate-dependent anesthesiologists returning to practice. J. Addict. Med. 2011, 5, 279-283. [CrossRef]

38. Angres, D.; Bologeorges, S.; Chou, J. A two year longitudinal outcome study of addicted health care professionals: An investigation of the role of personality variables. Subst. Abus. 2013, 7, 49-60. [CrossRef]

39. Cross, W.; Bologeorges, S.; Angres, D. Issues and data associated with addictive disease in pharmacists. Am. J. Addict. 2012, 21, 383.

40. Earley, P.H.; Zummo, J.; Memisoglu, A.; Silverman, B.L.; Gastfriend, D.R. Open-label study of injectable extended-release naltrexone (XR-NTX) in healthcare professionals with opioid dependence. J. Addict. Med. 2017, 11, 224-230. [CrossRef]

41. Bruguera, E.; Heredia, M.; Llavayol, E.; Pujol, T.; Nieva, G.; Valero, S. Integral Treatment Programme for Addicted Physicians: Results from the Galatea Care Programme for Sick Physicians. Eur. Addict. Res. 2020, 26, 122-130. [CrossRef]

42. Miller, W.R.; Walters, S.T.; Bennett, M.E. How effective is alcoholism treatment in the United States? J. Stud. Alcohol 2001, 62, 211-220. [CrossRef]

43. van Wamel, A.; Croes, E.; van Vugt, M.; van Rooijen, S. Prevalentie, Zorgaanbod, Effectiviteit en Trends in de Verslavingszorg. Achtergrondstudie in Opdracht van Het College van Zorgverzekeringen; Trimbos Insituut: Utrecht, The Netherlands, 2014.

44. NIDA. Treatment and Recovery: National Institute on Drug Abuse. 2020. Available online: www.drugabuse.gov/publications/ drugs-brains-behavior-science-addiction/treatment-recovery (accessed on 2 November 2020).

45. McKay, J.R.; Knepper, C.; Deneke, E.; O’Reilly, C.; DuPont, R.L. Corrigendum to "an initial evaluation of a comprehensive continuing care intervention for clients with substance use disorders: My first year of recovery (MyFYR)". J. Subst. Abus. Treat. 2017, 75, 97. [CrossRef] [PubMed]

46. McKay, J.R.; Knepper, C.; Deneke, E.; O’Reilly, C.; DuPont, R.L. An initial evaluation of a comprehensive continuing care intervention for clients with substance use disorders: My First Year of Recovery (MyFYR). J. Subst. Abus. Treat. 2016, 67, 50-54. [CrossRef] [PubMed]

47. Fazzino, T.L.; Bjorlie, K.; Lejuez, C.W. A systematic review of reinforcement-based interventions for substance use: Efficacy, mechanisms of action, and moderators of treatment effects. J. Subst. Abus. Treat. 2019, 104, 83-96. [CrossRef] [PubMed]

48. Kale, N. Urine Drug Tests: Ordering and Interpreting Results. Am. Fam. Phys. 2019, 99, 33-39.

49. Brooks, S.K.; Gerada, C.; Chalder, T. Doctors and dentists with mental ill health and addictions: Outcomes of treatment from the Practitioner Health Programme. J. Ment. Health 2013, 22, 237-245. [CrossRef] [PubMed]

50. Gross, S.R.; Wolff, K.; Strang, J.; Marshall, E.J. Follow-up of provision of inpatient treatment for UK healthcare professionals with alcohol dependence: Snapshot of a pilot specialist national health service. Subst. Use Misuse 2009, 44, 1916-1925. [CrossRef]

51. Johnson, R.P.; Connelly, J.C. Addicted physicians. A closer look. JAMA 1981, 245, 253-257. [CrossRef]

52. Kliner, D.J.; Spicer, J.; Barnett, P. Treatment outcome of alcoholic physicians. J. Stud. Alcohol 1980, 41, 1217-1220. [CrossRef]

53. Lloyd, G. One hundred alcoholic doctors: A 21-year follow-up. Alcohol Alcohol. 2002, 37, 370-374. [CrossRef]

54. Morse, R.M.; Martin, M.A.; Swenson, W.M.; Niven, R.G. Prognosis of physicians treated for alcoholism and drug dependence. JAMA 1984, 251, 743-746. [CrossRef]

55. Murray, R.M. Characteristics and prognosis of alcoholic doctors. Br. Med. J. 1976, 2, 1537-1539. [CrossRef] [PubMed]

56. Smith, P.C.; Smith, J.D. Treatment outcomes of impaired physicians in Oklahoma. J. Okla. State Med. Assoc. 1991, 84, 599-603. [PubMed]

57. Stuyt, E.B.; Gundersen, D.C.; Shore, J.H.; Brooks, E.; Gendel, M.H. Tobacco use by physicians In a physician health program, implications for treatment and monitoring. Am. J. Addict. 2009, 18, 103-108. [CrossRef] [PubMed]

58. Wilson, J.E.; Kiselanova, N.; Stevens, Q.; Lutz, R.; Mandler, T.; Tran, Z.V. A survey of inhalational anaesthetic abuse in anaesthesia training programmes. Anaesthesia 2008, 63, 616-620. [CrossRef]

59. Blodgett, J.C.; Maisel, N.C.; Fuh, I.L.; Wilbourne, P.L.; Finney, J.W. How effective is continuing care for substance use disorders? A meta-analytic review. J. Subst. Abus. Treat. 2014, 46, 87-97. [CrossRef]

60. Lawson, N.; Boyd, J.W. Physician health program outcome data should be viewed with caution. Judges J. $2018,57,36$.

61. Mumba, M.N.; Baxley, S.M.; Cipher, D.J.; Snow, D.E. Personal Factors as Correlates and Predictors of Relapse in Nurses With Impaired Practice. J. Addict. Nurs. 2019, 30, 24-31. [CrossRef]

62. Higgins, J.P.T.; Thomas, J.; Chandler, J.; Cumpston, M.; Li, T.; Page, M.J. Cochrane Handbook for Systematic Reviews of Interventions Version 6.0 (Updated July 2019): Cochrane. 2019. Available online: www.training.cochrane.org/handbook (accessed on 2 November 2020). 